CUAD. CONTAB. / BOCOTÁ, COLOMBIA, 17 (44): 377-420 / JULIO-DICIEMBRE 2016 / 377

\title{
Costos logísticos y metodologías para el costeo en cadenas de suministro: una revisión de la literatura*
}

doi:10.11144/Javeriana.cc17-44.clmc

\section{Javier Arturo Orjuela-Castro}

$\mathrm{PhD}$ (c) ingeniería - industria y organizaciones, Universidad Nacional de Colombia. MSc, investigación de operaciones y estadística, Universidad Tecnológica de Pereira, UTP. Especialista en ingeniería de producción, ingeniero industrial, Universidad Distrital Francisco José de Caldas. Ingeniero de alimentos. Docente e investigador en logística, Universidad Distrital Francisco José de Caldas. Líder del Grupo de Investigación de Cadenas de Abastecimiento, Logística y Trazabilidad, GICALyT.

Correo electrónico: jorjuela@udistrital.edu.co

\section{Norberto Suárez-Camelo}

Ingeniero industrial, Universidad Distrital Francisco José de Caldas. Integrante del Grupo de Investigación de Cadenas de Abastecimiento, Logística y Trazabilidad, GICALyT. Correo electrónico: norberto.suarez@live.com

\section{Yamit Israel Chinchilla-Ospina}

Ingeniero industrial, Universidad Distrital Francisco José de Caldas. Integrante del Grupo de Investigación de Cadenas de Abastecimiento, Logística y Trazabilidad, GICALyT. Correo electrónico: yamit.chinchillao@gmail.com

\footnotetext{
* Artículo científico, revisión de la literatura.
} 
Resumen Este artículo presenta una revisión de la literatura sobre las metodologías para el costeo en las cadenas de suministro (CS), con énfasis en costos logísticos. A partir de esbozar una perspectiva de análisis, evalúa las diferentes metodologías para medir el desempeño de la CS y la logística. Así mismo, establece en qué eslabón se aplica, si a toda la CS o a la empresa. Luego de determinar una taxonomía, expone de manera detallada los costos y procesos aplicados por los diferentes autores, también examina las diferencias fundamentales entre las metodologías. Se encuentra la necesidad de desarrollar una metodología propia para determinar los costos de la logística de medios y modos en la CS.

Palabras clave Costos logísticos; cadenas de suministro; metodologías; revisión literatura

Códigos JEL L90, M11, N76, Z00.

\section{Logistic Costs and Methodologies for Supply Chain Costing: a Literature Review}

\begin{abstract}
This article presents a literature review on the methodologies for supply chain (SC) costing, with an emphasis on logistics costs. Based on the outline of an analytical perspective, we evaluate the different methodologies for the measurement of SC performance and logistics. Likewise, we establish the link to which it is applicable, whether it is the whole CS or the company. After determining a taxonomy, we explain in detail the costs and processes applied by the different authors, while also examining the fundamental differences between methodologies. We find out there is a need to develop a methodology to determine the costs of the logistics of means and modes in SC.
\end{abstract}

Keywords Logistic costs; supply chains; methodologies; literature review

\section{Custos logísticos e metodologias para o custeio em cadeias de suprimentos: revisão de literatura}

Resumo Este artigo apresenta uma revisão de literatura sobre metodologias para custeio nas cadeias de suprimentos (CS), com destaque para os custos logísticos. A partir do esboço de uma perspectiva de análise, avalia as diferentes metodologias para medir o desempenho da CS e a logística. Mesmo, estabelece em qual elo é aplicado, se a toda a CS ou à empresa. Após determinar uma taxonomia, expõe detalhadamente os custos e processos aplicados pelos diferentes autores, mesmo que examina as diferencias principais entre metodologias. Acha-se a necessidade de desenvolver uma metodologia própria para determinar custos da logística de meios e modos na CS.

Palavras-chave Custos logísticos; cadeias de suprimentos; metodologías; revisão de literatura

\section{Introducción}

En el entorno económico moderno, las cadenas de suministro (CS) tienen fuentes de ventaja competitiva, como el valor agregado y los bajos costos de producción (Christopher, 2011; Mena, Whicker, Templar \& Bernon, 2002). Las actividades logísticas organizadas pueden derivar en la reducción de los costos totales, reducir los conflictos en operaciones internas y mejorar el servicio al cliente (Lambert, Stock \& Ellram, 1998). En Colombia, las CS agroalimentarias (CSA) buscan alternativas para su crecimiento económico; la logística en la CS ha generado ventajas competitivas, lo que requiere la evaluación de los costos para los diferentes agentes de la CS, productores, transformadores, comerciantes y distribuidores (Orjuela-Castro, Castañeda-Calderón \& Calde- 
rón, 2011). La reducción de los costos aumenta la rentabilidad y permite ofrecer menores precios al cliente (Bartolacci, 2004; Mena, Whicker, Templar \& Bernon, 2002; Seuring, 2002).

Falta información relacionada con el costo de sus operaciones (Everaert, Bruggeman, Sarens, Anderson \& Levant, 2008); identificar los costos logísticos requiere información precisa y específica (Pohlen \& LaLonde, 1994); las herramientas de análisis de costos no han tenido un desarrollo adecuado, los administradores del área logística necesitan clasificar los costos logísticos con criterios como su función, los territorios, las mercancías, los canales, el tamaño de las órdenes y el método de entrega, entre otros (Christopher, 2011; LaLonde \& Pohlen, 1996).

El objetivo de la revisión del estado del arte es proponer una taxonomía de los costos logísticos e identificar las metodologías y/o métodos propuestos para su medición en la CSA, y la relación con su desempeño. El documento está organizado en la metodología, los costos en la CS, los métodos y metodologías para la identificación y medición de los costos, los resultados y discusión, y las conclusiones.

\section{Metodología}

La revisión de la literatura en la temática de costos logísticos y CS se realizó en las bases Scopus, ScienceDirect, Google Scholar, IEEE Xplore, SpringerLink y ProQuest. Las fórmulas de búsqueda y selección fueron logistics cost, supply chain cost, agricultural products, food and drink, performance, cost accounting, cost management, accounting systems, entre los cuales se encuentran ABC (Activity-Based Costing), TCO
(Total Cost of Ownership), SCC (Supply Chain Costing) y SCTCM (Supply Chain Time-Cost Mapping).

La búsqueda fue guiada por las siguientes preguntas: ¿cuál ha sido el desarrollo de la disciplina con el paso de los años?, ¿qué diferencia hay entre el costo de la CS y los costos logísticos?, ¿cuáles son los sistemas de costeo más utilizados en la CS?, ¿cuáles son los beneficios de tener una metodología para la identificación de los costos logísticos?, ¿hay metodologías de costos enfocadas en las CSA y/o de alimentos perecederos?

Una primera búsqueda permitió observar la evolución de la temática de costos logísticos en la CS; entre 2004 y 2008, está la mayor cantidad de publicaciones; luego creció hasta 2014 (gráfica 1).

Una segunda búsqueda más específica permitió identificar las publicaciones referentes a costos logísticos y su relación con sistemas de costeo en CS, de las que se escogieron 146 artículos relacionados con costos logísticos y metodologías de costeo en CS. La gráfica 2 muestra los años de mayor publicación con una tendencia creciente en las últimas décadas.

\subsection{Costos logísticos y su relación con el desempeño}

La perspectiva de análisis definida para el desarrollo de este artículo considera la relación existente entre los costos logísticos y el desempeño de la CS, y la interacción entre los componentes del costo logístico y las metodologías de identificación de costos. Analiza las medidas de desempeño en la CS en complemento con el costo logístico para los distintos modos y medios. 


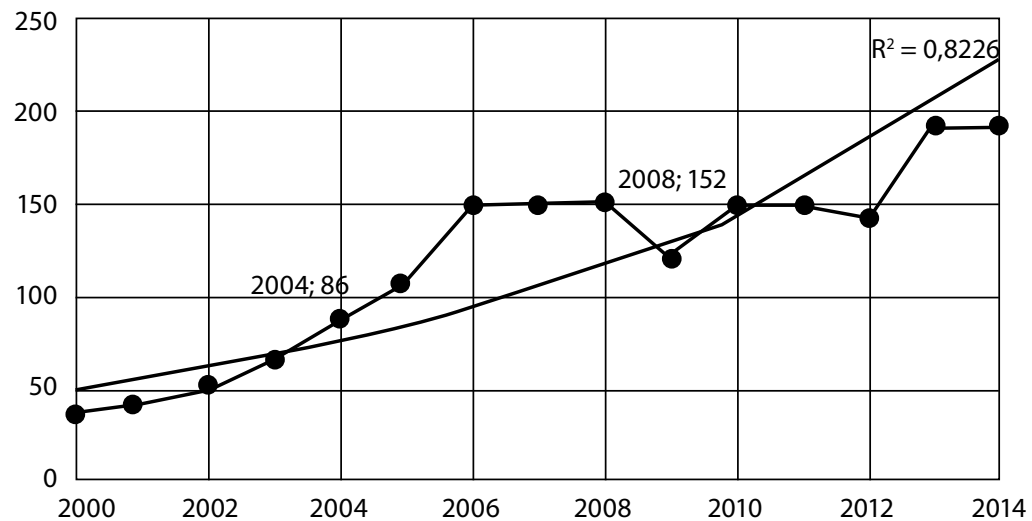

Gráfica 1

Evolución histórica de la temática de costos logísticos

Fuente: elaboración propia, a partir de las bases de datos consultadas

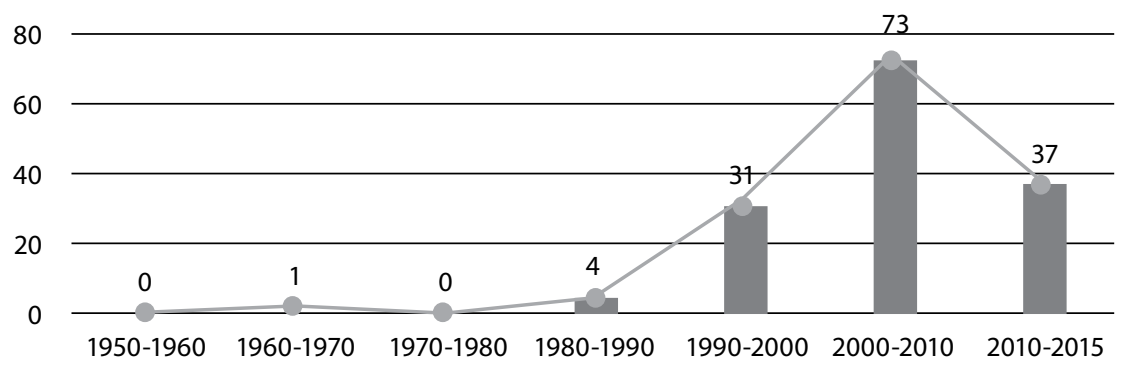

\section{Gráfica 2}

Cronología de publicaciones en metodologías de costeo y su relación con los costos logísticos Fuente: elaboración propia, a partir de las bases de datos consultadas

\section{Costos logísticos en la cadena de suministro}

La operación de los sistemas logísticos involucrados en la CS requiere recursos, los cuales varían según el tipo y tamaño de la empresa, los productos, los canales de distribución y las características del cliente.

En el ámbito logístico, los costos se relacionan con "un grupo de costos adheridos a las funciones de la empresa, que gestionan y controlan los flujos de materiales y sus flujos de información y agrupan todos los costos adheridos a las actividades de la empresa" (Pau i Cos \& De Navascués y Gasca, 1998).

La gráfica 3 presenta la taxonomía definida para el desarrollo del artículo. De una parte, se establecieron las metodologías encontradas; de otra, las medidas de desempeño tanto para la logística como para la CS. 


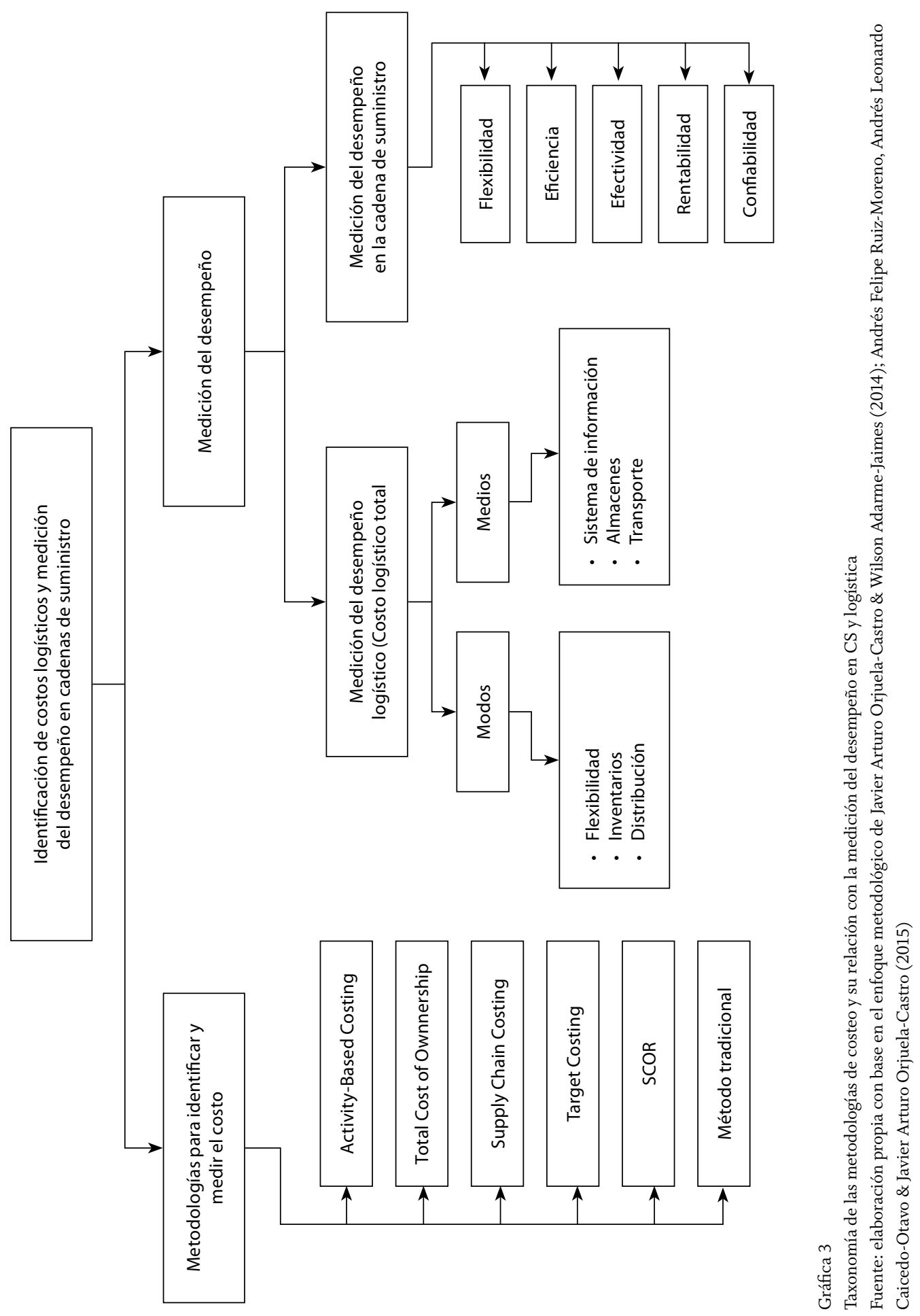




\subsubsection{Relación de los costos con el desempeño}

Las operaciones logísticas incluidas para determinar el costo en la CS incluyen las relacionadas con el flujo y almacenamiento del producto desde el productor de materias primas hasta el consumidor final. Donald J. Bowersox, David J. Closs y Bixby Cooper (2007), Carlos F. Daganzo (2005), Mikel Mauleón-Torres (2006), Sahidah Zakariah y Jaafar Pyeman (2013) recomiendan profundizar en los costos logísticos como guía para gestionar y mejorar su propia rentabilidad y mejorar la confiabilidad.

Los costos logísticos permiten la cuantificación en unidades monetarias del uso de recursos empleados en una actividad o proceso logístico. La gestión contable utiliza términos incompletos para la medición de los costos de los procesos logísticos o de la cadena, pues no desagrega cada actividad ni tiene en cuenta los enlaces de la CS.

Medir el costo logístico total mejora el rendimiento financiero de la CS, permite tomar decisiones con una visión más amplia, su medición correcta brinda información para una mejor implementación de estrategias sobre el flujo de materiales e información asociada en cada eslabón (Bhagwat \& Sharma, 2007). Los costos logísticos permiten determinar el rendimiento sobre el capital invertido y el retorno de la inversión, el cual es directamente proporcional el rendimiento logístico de la CS.

\subsubsection{Costos de aprovisionamiento}

El proceso de aprovisionamiento comprende gestión de proveedores y de compras. Comprende actividades como la selección de pro- veedores; la negociación de precio, términos y cantidades; la generación de órdenes para compras y el control de pedidos (Ballou, 2004; Gudehus \& Kotzab, 2009; Lambert, Stock \& Ellram, 1998). Ronald H. Ballou (2004) propone once (11) actividades básicas. Timm Gudehus y Herbert Kotzab (2009) establecen ocho (8) competencias que deben desarrollarse en la empresa para una correcta gestión de compras.

El costo total de aprovisionamiento incluye los costos asociados a la gestión de proveedores (Frazelle, 2002) y de compras (Kivinen \& Lukka, 2004). Donald J. Bowersox, David J. Closs y Bixby Cooper (2007) proponen una segmentación exhaustiva de los costos relacionados con la gestión de pedidos. Recientemente, se ha propuesto un costeo basado en el costeo por actividades ABC aplicado a compras y aprovisionamiento, denominado costeo total de propiedad (TCO, Total Cost of Ownership) (Ellram, 2006b; Ellström, Rehme, Björklund \& Aronsson, 2012; Mauleón-Torres, 2006).

\subsubsection{Costo de gestión de inventarios}

La formulación de políticas relacionadas con el inventario tiene como propósito equilibrar el aprovisionamiento con la demanda, proteger frente a la incertidumbre de la demanda y los ciclos de pedido, y actuar como amortiguador entre las interfaces críticas del canal de suministro (Lambert, Stock \& Ellram, 1998); su adecuada utilización mejora la rentabilidad (Robeson \& Copacino, 1994).

Si bien mantener inventarios tiene un costo, usarlos puede reducir indirectamente los costos de otras actividades de la cadena (Ballou, 2004). Una importante porción del capital 
de trabajo de la empresa está bloqueada en el inventario. Si el inventario es excesivo en relación con el nivel óptimo, gran cantidad de fondos estarán aislados y no podrán ser usados en otros propósitos productivos, los fondos estarían atados innecesariamente (Sople, 2007).

Las medidas para reducir los costos relacionados con el inventario incluyen la reducción del número de pedidos pendientes o envíos urgentes, la eliminación de inventario muerto u obsoleto, o mediante el mejoramiento en la precisión de los pronósticos. El traslado de inventarios entre almacenes y los traslados de lotes pequeños pueden ser reducidos o eliminados con una mejor planeación del inventario (Ballou, 2004; Bowersox, Closs \& Cooper, 2007; Lambert, Stock \& Ellram, 1998).

Dadas las diferencias entre el entorno de las compañías, cada una de ellas posee su propia combinación de costos de inventarios; sin embargo, se pueden categorizar en costos de capital, del servicio de inventarios, de espacio y de riesgos asociados a inventarios (Lambert, Stock \& Ellram, 1998). Es posible establecer dos categorías relacionadas con el mantenimiento de inventarios: costos de posesión y de no posesión (Mauleón-Torres, 2006).

\subsubsection{Costos de almacenamiento}

El almacenamiento se puede definir como el componente del sistema logístico de la empresa que guarda productos (materias primas, partes, productos en proceso y productos terminados) en (y entre) los puntos de origen y de consumo. El almacenamiento tiene tres funciones básicas: movimiento, depósito y transferencia de información (Lambert, Stock \& Ellram, 1998).
El costo relacionado con el almacenaje está compuesto por los costos de espacio, de las instalaciones, manipulación y de tenencia del inventario (Pau i Cos \& Navascués y Gasca, 1998). Estos costos se generan al realizar las funciones básicas del almacenamiento como mantenimiento o pertenencia, consolidación de productos, carga fraccionada y/o mezcla (Ballou, 2004). Annelie Petterson y Anders Segerstedt (2012) los dividen en costo de inspección de bienes entrantes, del personal que trabaja en el almacén y por el edificio. La distribución de los costos en un almacén convencional corresponde a un $50 \%$ en preparación de pedidos o picking, 20\% por almacenamiento, $15 \%$ por recepción y 15\% por envío (Frazelle, 2002).

Hanne-Mari Hälinen (2015) establece la diferencia entre costo de almacenamiento y costo de gestión de inventarios; el primero varía en función de la cantidad de instalaciones y su operación, el segundo en relación con el nivel de inventario o unidades en existencia. En contraste, el costo de espacio de almacenamiento proporcionado por proveedores de servicios logísticos tercerizados se asigna a costo de mantenimiento de inventario, ya que típicamente se cargan con base en el volumen de las mercancías (Zakariah \& Pyeman, 2013).

\subsubsection{Costo de distribución}

Estos costos consideran toda actividad involucrada en el movimiento de bienes y materiales, el volumen de la carga, el peso de la carga, la distancia y los puntos de origen y de destino, entre otros factores (Lambert, Stock \& Ellram, 1998). Encuestas realizadas por Hanne-Mari Hälinen (2015), Yung-yu Tseng, Wen Long Yue y Michael 
Taylor (2005), Sahidah Zakariah y Jaafar Pyeman (2013) han mostrado que este costo abarca un mayor porcentaje del costo total y es la categoría más importante de costo logístico. El costo del transporte incluye los medios, contenedores, paletas, terminales y tiempo (Hälinen, 2015; Tseng, Yue \& Taylor, 2005; Zakariah \& Pyeman, 2013). El costo de transporte se puede clasificar en costo de entrada que se asocia a las actividades de aprovisionamiento y el costo de salida asociado con la entrega de productos hacia los clientes finales (Parra-Ortega, 2010).

Los costos en los procesos de distribución se relacionan con el producto, el mercado, la capacidad, la distancia recorrida, el volumen transportado, la frecuencia y las rutas de distribución (Abdallah, 2004; Lambert, Stock \& Ellram, 1998). Con el producto, se relacionan con la densidad, facilidad y responsabilidad de almacenamiento y manejo. En el mercado se evalúan el grado de competencia, la ubicación, las regulaciones gubernamentales, el balance del tráfico de mercancías, la estacionalidad de los productos y si el comercio es nacional o internacional. Por su parte, la capacidad depende del número, el tipo y la disponibilidad del vehículo y los conductores. Mientras en la distancia, los factores más relevantes son los días requeridos para el transporte y el trayecto entre los centros de distribución (Abdallah, 2004).

En el proceso de distribución, los autores han reportado diferentes tipos de costos relacionados con los vehículos, los kilómetros recorridos y los días totales (Abdallah, 2004; Frazelle, 2002; Gudehus \& Kotzab, 2009).

Los costos relacionados con la carga dependen del modo utilizado, terrestre, férreo, aéreo, fluvial o marítimo y del servicio a la carga generado por agentes como las asociaciones de transportistas, las compañías de mercadeo intermodal, los proveedores de servicios logísticos y las compañías de envíos (Lambert, Stock \& Ellram, 1998).

\section{Métodos y metodologías para la identificación y medición de costos}

Las características de los sistemas logísticos implican el análisis de la CS y sus parámetros asociados. Los métodos tradicionales de costeo no ayudan a identificar del impacto a gran escala de las decisiones tomadas y muestran solamente el comportamiento de las áreas individuales (Christopher, 2011).

\subsection{Costeo tradicional}

Hay dos tipos de costeo tradicional, el costeo absorbente (Sinisterra-Valencia \& PolancoIzquierdo, 2007) y el costeo variable o directo (Amirkulova, 2011).

El costeo tradicional utiliza medidas relacionadas con el volumen, como la mano de obra; se basa en las unidades producidas para calcular las tasas de asignación de los gastos indirectos; y asigna los gastos indirectos en dos etapas, primero a una unidad organizacional ya sea la planta o algún departamento y luego a puestos de transformación (Quiñónez, González, López \& Tabares, 2005).

\subsection{Costeo basado en actividades (ABC, Activity-Based Costing)}

El sistema de costos basado en actividades, también conocido como ABC (Activity-Based 
Costing), surgió en los años 60 de manera incipiente y su auge se traslada a los años 80 (Gosselin, 2007). Se puede observar la evolución de la metodología cronológica. El costeo $\mathrm{ABC}$ tiene ventajas como la capacidad de administrar de manera adecuada los costos y las actividades, lo que llevó el sistema a emerger en el ámbito de la investigación (Gosselin, 2007).

Jeffrey G. Miller y Thomas E. Vollmann fueron los primeros en asignar los costos de transacción a procesos y afirmaron lo adecuado de controlar los costos indirectos como parte del estudio de los costos y las transacciones que los originan (Miller \& Vollmann, 1985). Los primeros en introducir la técnica $\mathrm{ABC}$ fueron Robin Cooper y Robert S. Kaplan (1988), que asignaron los costos indirectos a los productos o a cualquier objeto que haga parte de las actividades económicas (Bustamante-Salazar, 2014).

Ronald W. Hilton y David E. Platt (2005 p. 786) definen el modelo de administración basado en actividades (ABM, Activity-Based Management) ABM como: "El uso de un sistema de costeo basado en actividades para mejorar las operaciones de una organización”. Hilton y Platt (2005) plantean el procedimiento en dos etapas, "en la primera etapa, las actividades importantes son identificadas y los costos asignados a las actividades (fuentes) de acuerdo con los recursos consumidos por ellas. En la segunda etapa, los costos son asignados de cada actividad fuente de costo a las diferentes líneas de productos en proporción a la cantidad de elementos de costos consumidos por la línea de producto" (Marques, 2012).

Mike Walker (1998) propone un costeo basado en atributos, un método derivado del
$\mathrm{ABC}$, que proporciona un análisis detallado de coste-beneficio de las necesidades del cliente. Desagrega las necesidades de los clientes en atributos y enfoques de productos específicos sobre la planificación en lugar de analizar los costos pasados (Gosselin, 2007).

La herramienta $\mathrm{ABC}$ ha sido aplicada en múltiples contextos con diferentes resultados. En la tabla 1 se puede ver la gran cantidad de aplicaciones que tiene la metodología en diferentes sectores con características propias en cada uno. El desarrollo más reciente en $\mathrm{ABC}$ es el ABC Impulsado por Tiempo (TDABC, Time Driven Activity Based Costing), propuesto por Robert S. Kaplan \& Stephen R. Anderson (2004).

Bajo el sistema $\mathrm{ABC}$, los costos son clasificados en grupos de costos por actividad. Los factores de recursos son medidas del consumo en cada actividad y grupo de costos. Los factores de actividad son medidas del consumo por cada unidad de producto (Cooper \& Kaplan, 1991; Gosselin, 2007; Noreen, 1991; Pirttilä \& Hautaniemi, 1995).

A partir de Zhou Chaoyang y Jiang Ying (2010), Gary Cokins (2006), Patricia Everaert, Werner Bruggeman, Gerrit Sarens, Steven R. Anderson e Yves Levant (2008), María Rosa López-Mejía, Alicia Gómez-Martínez y Salvador Marín-Hernández (2011), Timo Pirttilä \& Petri Hautaniemi (1995), se pueden establecer una serie de etapas para determinar los diferentes costos de las operaciones logísticas de una empresa o en la CS con el método $\mathrm{ABC}$ :

- Identificación y clasificación de los principales procesos logísticos

- Descomponer los procesos en actividades 


\section{6 / VOL. 17 / NO. 44 / JULIO-DICIEMBRE 2016}

- Identificar los recursos consumidos en el desarrollo de las actividades

- Determinar los costos de las actividades mediante los factores de uso de recursos
- Asignar los costos relacionados con los productos por medio del factor de uso de las actividades

- Determinar el costo total

\section{Sector de aplicación Artículo}

María Rosa López-Mejía, Alicia Gómez-Martínez \& Salvador Marín-Hernández

(2011)

Patxi Ruiz de Arbulo-López, Jordi Fortuny-Santos, Carla Vintró-Sánchez \& Aitor Basáñez-Llantada (2013)

Industria manufacturera

Servicios públicos (energía, agua)

Instituciones financieras

Sector público

Comestibles/Alimentos

Seguros de vida

Hospitales

Operadores logísticos

Universidades e instituciones educativas
Manuel Schulze, Stefan Seuring \& Christian Ewering (2012)

Radu Mariana (2013)

Figen Öker \& Hümeyra Adigüzel (2010)

Diobaldo César Heredia-Gutiérrez (2010)

Julijana Krajnc, Klavdij Logožar \& Bojana Korošec (2011)

John L. Eggers \& Charles E. Bangert (1998)

Maria Letiţia Rof \& Nicoleta Farcane (2011)

John Innes \& Falconer Mitchell (1997)

Trond Bjørnenak (2000)

Michael Garry (1996)

Tom Groot (1999)

Timo Pirttilä \& Petri Hautaniemi (1995)

Jing Zang, Keming Z. Zhang \& Rui Zhao (2008)

Mike Adams (1996)

Margaret King, Irvine Lapsley \& Falconer Mitchell (1994)

Brian Aird (1996)

Luis Fernando Gómez-Montoya, María Isabel Duque-Roldán \& Jamer CarmonaLópez (2009)

Peng Zheng \& Man Wang (2011)

Isabel Hall Themido, Amílcar Arantes, Carla Fernandes \& A. Paulo Guedes (2000)

Sirirat Somapa, Martine Cools \& Wout Dullaert (2010)

Maria da Conceição da Costa Marques (2012)

Tabla 1

Aplicaciones de la metodología ABC

Fuente: elaboración propia con base en la revisión de la literatura 
Con base en la determinación y análisis de las actividades logísticas realizadas y su respectivo consumo y su aporte al desarrollo de los productos finales de la empresa, es posible tomar decisiones para mejorar el desempeño logístico, mediante la eliminación, selección o reducción de actividades (Chaoyang \& Ying, 2010).

\section{Como avance y complemento del $\mathrm{ABC}$ se} desarrolló el Time Driven Activity-Based Costing (TDABC). Este sistema permite asignar los recursos directamente a los objetos de costos, mediante un coeficiente de capacidad, se calcula dividiendo el costo de los recursos sobre su capacidad práctica, es decir, el tiempo que estos están disponibles en la organización, para su posterior asignación a los objetos de costo (Bustamante-Salazar, 2014; Kaplan \& Anderson, 2004). Al calcular la capacidad y dividirla por el costo total de la operación del departamento, se obtiene el costo por unidad de tiempo de operación de determinado departamento. Basado en esto se estima el tiempo de desarrollar determinada actividad según sus características (Everaert, Bruggeman, Sarens, Anderson \& Levant, 2008; Somapa, Cools \& Dullaert, 2010).

\subsection{Costeo total de propiedad (TCO, Total Cost of Ownership)}

El costo de la propiedad, planteado por Lisa M. Ellram (1995), introdujo el TCO como herramienta para la compra en la empresa, con el objetivo de establecer el costo de la relación con los proveedores.

Hay múltiples definiciones de TCO. Frederik Zachariassen y Jan Stentoft (2011) citan una serie de definiciones de múltiples autores. El TCO considera una aplicación del método
ABC que dictamina los costos del ciclo completo de compras o adquisición de la cantidad de productos, permite la comprensión del costo de realizar negocios con un proveedor particular (Degraeve, Labro \& Roodhooft, 2000; Ellram, 1995; Garfamy, 2006; Wouters, Anderson \& Wynstra, 2005).

El modelo TCO identifica y cuantifica todos los costos asociados con el proceso de compras a lo largo de toda la cadena de valor. El modelo relaciona los costos con el elemento, el servicio, la calidad, las entregas, la administración, la comunicación, las fallas y el mantenimiento, entre otras fuentes (Degraeve, Labro \& Roodhooft, 2000). Para incluir todas las fuentes de costos durante el ciclo de vida del producto, Lisa M. Ellram (2006b) desarrolló un diagrama de flujo de actividades con tres grupos: pretransacción, transacción y postransacción que, a su vez, están divididos en cuatro subgrupos: componentes y materiales, bienes de capital, mantenimiento y servicios.

Hay múltiples beneficios al aplicar TCO en una compañía: evaluación consistente de proveedores, medición del desempeño de proveedores, selección de proveedores, determinación de cantidades a ordenar, evaluación de las opciones estratégicas de compra, mejorar las negociaciones con proveedores, control de riesgo, incorporación de elementos no relacionados con el precio en las decisiones de compra (Bremen, Oehmen \& Alard, 2007).

Los avances que ha tenido la metodología TCO desde su creación y los aportes de los autores relacionados son enunciados por Federico Caniato, Stefano Ronchi, Davide Luzzini y Olimpio Brivio (2015). Diversos autores han 
elaborado estudios relacionados con el TCO, con diferentes contribuciones al incorporar esta metodología en distintos escenarios de bienes y servicios, proveedores o cadenas completas, uno o varios períodos, uno o múltiples proveedores (tabla 2).

\begin{tabular}{ll}
\hline \multicolumn{1}{c}{ Sector de aplicación } & \multicolumn{1}{c}{ Artículo } \\
\hline & Federico Caniato, Stefano Ronchi, Davide Luzzini y Olimpio Brivio (2015) \\
& Nicole Radziwill \& Ronald F. DuPlain (2010) \\
Industria manufacturera & Stefania Castellani, Antonietta Grasso, Jacki O'Neill y Peter Tolmie (2005) \\
& Roel J. Ritsma, André Tuyl \& Bas Snijders (2009) \\
Servicios públicos (Energía, agua) & Su Jiang (2013) \\
Múltiples sectores & Lisa M. Ellram (2006a) \\
Operadores logísticos & Cathy Macharis, Philippe Lebeau, Joeri van Mierlo \& Kenneth Lebeau (2013) \\
\hline
\end{tabular}

Tabla 2

Aplicación de la metodología TCO

Fuente: elaboración propia con base en la revisión de la literatura por Federico Caniato, Stefano Ronchi, Davide Luzzini y Olimpio Brivio (2015)

\subsection{Costo de la cadena de suministro (SCC, Supply Chain Costing)}

El SCC abarca desde el costo de producción del bien, la administración y el almacenamiento, hasta la distribución y el costo de capital (Petterson \& Segerstedt, 2012). El SCC incluye costos de transacción, información, flujo físico y de mantenimiento de inventario. Para asegurar que la administración de la CS cumpla sus objetivos, los SCC deben ser conocidos. Antes de establecer estos costos mediante el modelo $\mathrm{ABC}$ se deben conocer las actividades desarrolladas y su relación con el modelo de costeo (Lin, Collins \& Su, 2001; Lin, 2009).

- La metodología SCC involucra seis pasos (LaLonde \& Pohlen, 1996):

- Análisis de los procesos de la CS

- Descomposición de los procesos en actividades
- Identificación de los recursos requeridos para desarrollar una actividad

- Costear las actividades

- Rastreo de costos de las actividades hacia las salidas de la CS

- Análisis final. El SCC provee una herramienta al final para simular los inductores de costos de actividades específicas, la variación con el flujo del producto y la demanda que afecta el costo a lo largo de la CS.

El SCC emplea en su estructura técnicas integradas como rentabilidad directa del producto (DPP, Direct Product Profitability), costeo basado en actividades (ABC, Activity Based Costing), costeo total de propiedad (TCO, Total Cost of Ownership) y respuesta eficiente al consumidor (ECR, Efficient Consumer Response); sin em- 
bargo, difieren según las actividades a lo largo de la cadena (LaLonde \& Pohlen, 1996).

\subsection{Análisis de cadena de valor}

\section{(AV, Value Analysis)}

La forma en que se relacionan los procesos y la administración moderna ha generado nuevos desafíos en la contabilidad empresarial. Se requiere optimizar y coordinar de manera adecuada el flujo de información a lo largo de la cadena de valor; una metodología adecuada con este propósito es el AV (Dekker, 2001). Este modelo útil para analizar las fuentes de ventajas competitivas de una empresa, fue desarrollado por Michael Porter en los años 80 (Orjuela-Castro, Castañeda-Moreno, Canal-Roa \& Rivera-Velasco, 2015). Michael Porter afirma que hay que reconocer en las cadenas productivas los proveedores, compradores y canales como aliados en una competencia internacional y no solo como componentes de una transacción, con dos enfoques: la generación de productos y el desarrollo de sistemas de gestión (Porter, 1990). La definición de cadena de valor se ha extendido con el paso de los años, Duruflé, citado en Orjuela, la define como el conjunto de agentes económicos que contribuyen directamente a la producción, procesamiento y distribución hasta el mercado de un determinado producto (Duruflé, 1993, citado en Orjuela-Castro, Castañeda-Calderón \& Calderón, 2011). John K. Shank y Vijay Govindarajan definen la cadena de valor como el conjunto de actividades que crean valor en la cadena desde las fuentes de material o proveedores hasta que se convierte en producto terminado y llega a las manos del consumidor final (Shank \& Govindarajan, 1993).
La evolución de la metodología de AV se presenta en la gráfica 4. Michael Porter (1990) propuso el concepto de valor de la cadena y un modelo de cadena de valor como medio de análisis para la competitividad; Keivan Zokaei parte de los estudios desarrollados por Michael Porter, John K. Shank y Vijay Govindarajan, Keivan Zokaei y David W. Simons, entre otros, para elaborar una metodología que permita crear valor con base en la cultura de lean manufacturing, teniendo como resultado una extensión de la cadena de valor, modelado a toda la CS, para el análisis de competitividad entre empresas (Zokaei \& Simons, 2006).

El concepto de AV en la administración de la contabilidad fue introducido por John K. Shank y Vijay Govindarajan (1993). La idea central del $\mathrm{AV}$ es romper la CS en actividades que ocurren desde los materiales básicos de fabricación hasta el cliente final en segmentos estratégicos relevantes para entender el comportamiento de los costos y las características que lo diferencian de otra cadena (Dekker, 2001; Suárez, 2013). Keivan Zokaei (2007) afirma que el AV es muy diferente al análisis en la CS, dado que su concepto enfatiza en la orientación al cliente y el valor percibido por este; este análisis se enfoca en la efectividad de la CS. El AV se convierte entonces en un método para analizar los efectos de actividades estratégicas en el costo y las relaciones de la cadena de valor. Alexandra van den Abbeele, Filip Roodhooft y Luk Warlop, citados por Mustafa Kirli y Harun Gümüş, afirman que el AV se puede usar para analizar, coordinar y optimizar los enlaces entre las actividades en la cadena de valor, pero enfocándose en la interdependencia de estas (Kirli \& Gümüş, 2011). 
Se pueden distinguir diferentes relaciones en la cadena de valor: entre actividades, entre eslabones y entre la empresa sus proveedores y clientes (Dekker, 2001). Mustafa Kirli y Harun Gümüş (2011) aseguran que las empresas pueden agrupar las actividades en nueve grupos al relacionar actividades con los proveedores, canales y consumidores finales. Cinco de estas actividades se consideran primarias y están relacionadas directamente con lo concerniente a crear los productos, comercializarlos y entregarlos. Los cuatro grupos restantes de actividades son de soporte, están repartidos a través de las actividades primarias, y se pueden usar para incrementar la efectividad y la eficiencia.

En la tabla 3 se pueden identificar las aplicaciones de AV. La metodología de AV y el sistema de valores son puntos de vista basados en la actividad de la empresa y la cadena. Un mayor detalle de la metodología para la aplicación de análisis de valor se puede consultar en Mustafa Kirli y Harun Gümüş (2011) e Ibrahim Abd El Mageed y Ali El Kelety (2006).

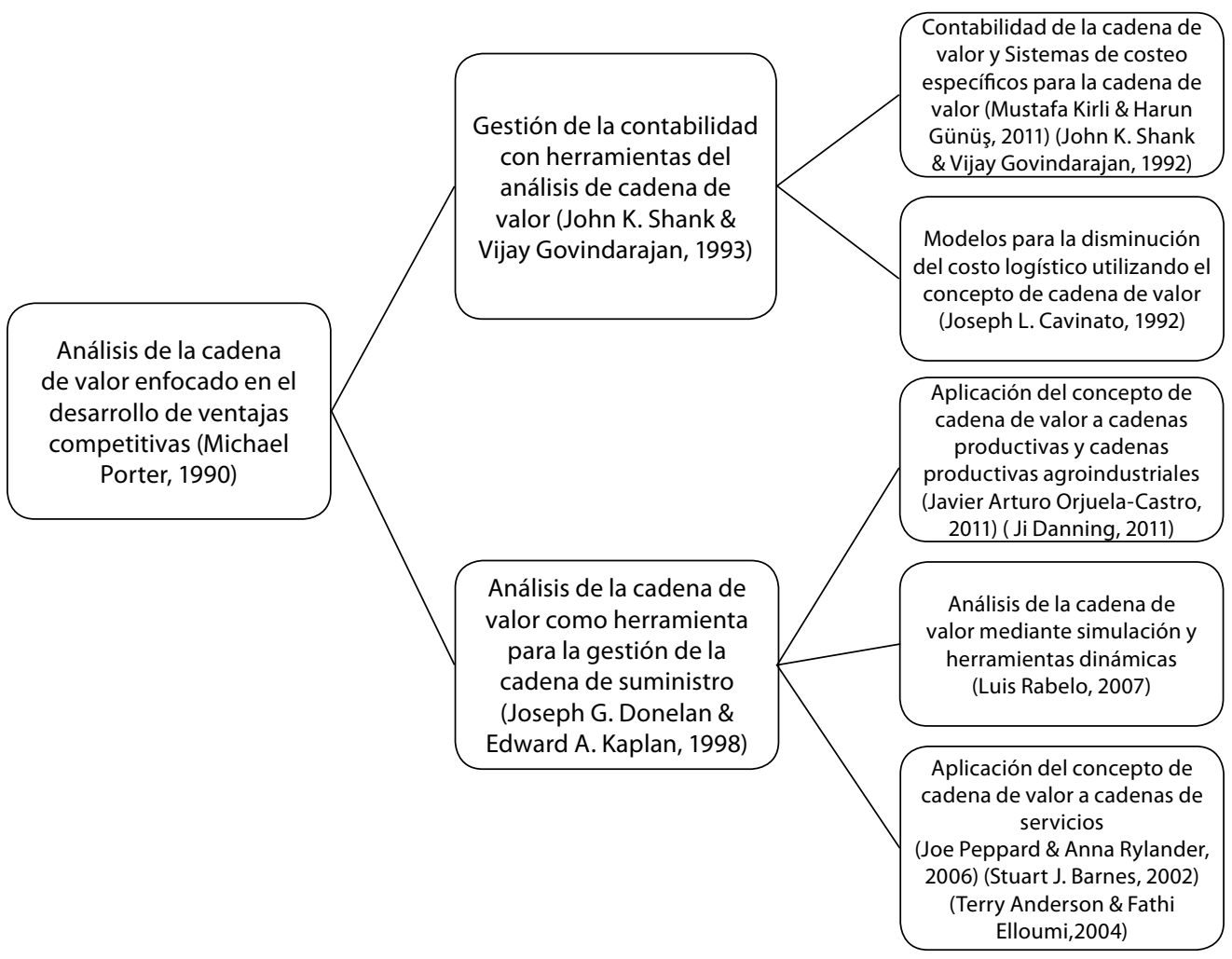

Gráfica 4

Evolución histórica de la metodología Análisis de cadena de valor Fuente: elaboración propia con base en la revisión de la literatura 
costos logísticos Y METOdolocías PARA EL COSTEO EN CADENAS / J. ORJUELA, N. SUÁREz, Y. CHINCHILLA / 391

\begin{tabular}{ll}
\hline \multicolumn{1}{c}{ Campo de utilización } & \multicolumn{1}{c}{ Autor } \\
\hline Contabilidad & Henri C. Dekker (2001), Mustafa Kirli \& Harun Gümüş (2011) \\
Innovación & Michael Porter (1990) \\
Agroindustria & Ji Danning (2011), Javier Arturo Orjuela-Castro, Camilo Andrés Castañeda-Calderón \\
& \& María Eugenia Calderón (2011) \\
Consumo masivo & Dian R. Setyawati, Imam Santoso \& Mas'ud Effendi (2012) \\
Costeo en cadena de valor & (Shank \& Govindarajan, 1993) \\
Tecnologías de la información & Stuart J. Barnes (2002), Terry Anderson \& Fathi Elloumi (2004) \\
\hline
\end{tabular}

Tabla 3

Aplicaciones de la metodología Análisis de cadena de valor

Fuente: elaboración propia con base en la revisión de la literatura

En el caso de las cadenas agroalimentarias, el análisis de la cadena de valor se refiere a toda la cadena vertical de actividades, desde la producción en el establecimiento agropecuario pasando por la etapa de procesamiento y por la distribución mayorista y minorista (OrjuelaCastro, Castañeda-Calderón \& Calderón, 2011). La cadena de valor integra el aprovisionamiento, almacenamiento, distribución y otras fases antes del consumo (Danning, 2011).

\subsection{Costeo de cadena de valor (VCC, Value Chain Costing)}

En la gestión de la contabilidad, el costo de la cadena de valor es una herramienta analítica de gestión estratégica de la contabilidad (Dekker, 2001). John K. Shank y Vijay Govindarajan (1993) desarrollaron un método para costear la cadena de valor (VCC), basado en el análisis propuesto por Michael Porter (1990) y Mustafa Kirli y Harun Gümüş (2011). Esta técnica se enfoca en el exterior de las organizaciones como la clave para la creación de valor en las actividades asociadas a la manufactura del producto. El VCC propone un acercamiento a la contabilidad que considera todas las actividades desde el diseño hasta la distribución del producto. Las implicaciones estratégicas con respecto a la economía y la eficiencia se derivan de los enlaces entre empresas, proveedores y clientes. El VCC ha sido usado como estrategia de gestión de la contabilidad, y tiene en cuenta el costo localizado en las actividades de diseño, aprovisionamiento, producción, fabricación, distribución y la logística de servicio del producto (Kirli \& Gümüş, 2011; Shank \& Govindarajan, 1993).

\subsection{Costeo por objetivos (TC, Target Costing)}

Con el incremento de los competidores en un mercado mundial, las compañías han optado por buscar que sus productos tengan el mayor valor posible al menor costo. El costo objetivo (TC) es el proceso que permite alcanzar el precio que el mercado puede soportar. El TC reduce el costo de los nuevos productos para alcanzar el nivel requerido de ganancia, satisfacción, calidad, tiempo de entrega, desarrollo del producto a tiempo (Lin, 2009).

El TC es un modelo de reducción del costo y un sistema de control usados para disminuir el costo del producto durante las etapas tempranas 
de su ciclo de vida. El método difiere de los métodos tradicionales de costeo, ya que ayuda a fijar el precio de venta y permite controlar el costo a tiempo en varias eslabones de la cadena. El TC planifica de manera proactiva el costo y lo reduce al gestionar desde las primeras etapas de diseño y desarrollo (Institute of Management Accountants, 1994)

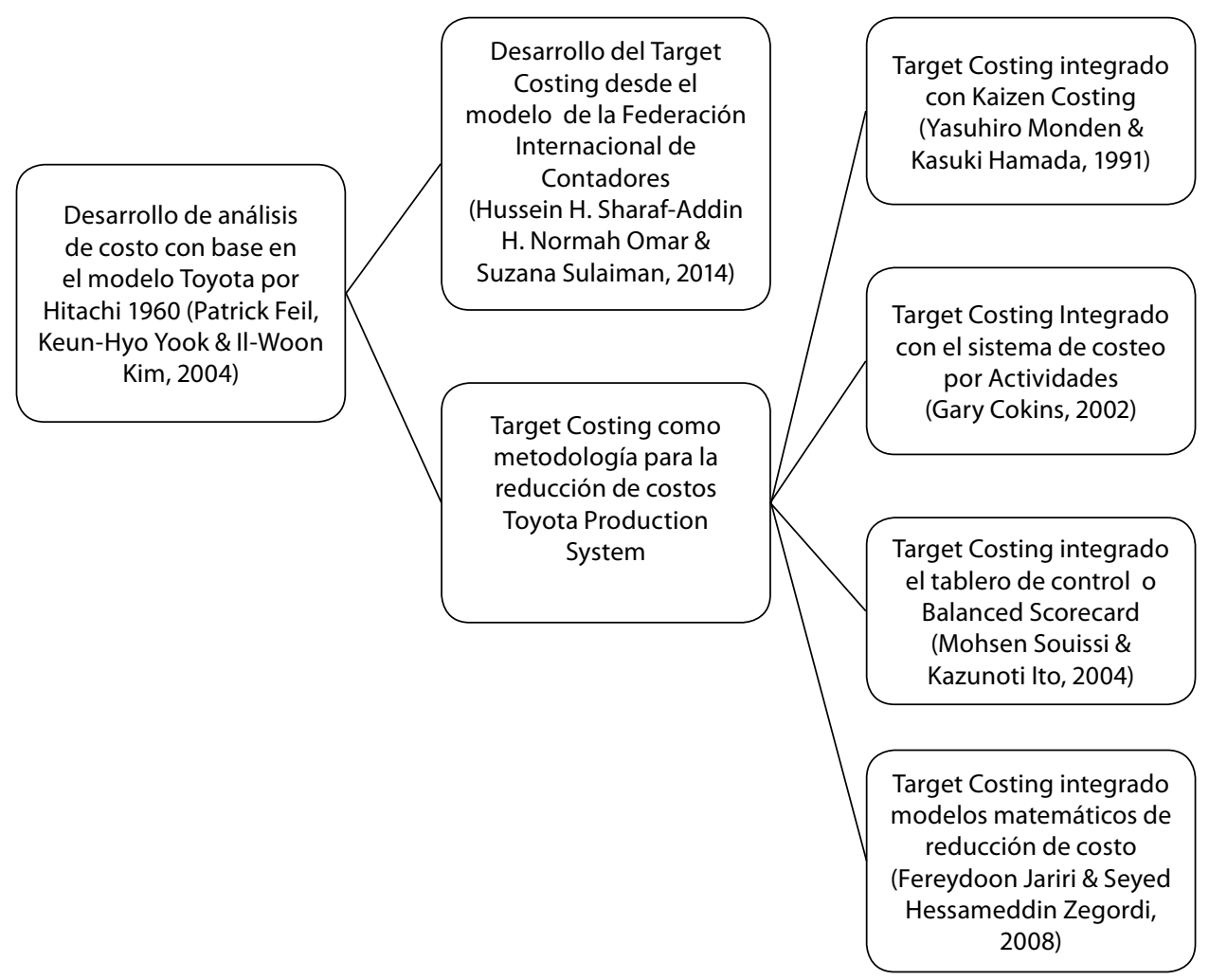

Gráfica 5

Desarrollo cronológico de la metodología de Target Costing

Fuente: elaboración propia con base en la revisión de la literatura

En la gráfica 5 se presenta el desarrollo cronológico de la metodología TC. Robin Cooper y Regine Slagmulder (1997) definieron el TC como una técnica de gestionar futuras ganancias en la organización, que empieza con el precio objetivo, el cual es determinado por investigaciones de mercado u observaciones: el beneficio deseado será entonces la resta del precio obje- tivo menos el costo objetivo (Filomena, Neto \& Duffey, 2009; Sharaf-Addin, Omar \& Sulaiman, 2014). El TC emerge en Japón como una técnica de gestión de los contadores para tomar mejores decisiones durante los procesos de planeación y desarrollo de un producto, y estimular a los empleados a proponer estrategias (Filomena, Neto \& Duffey, 2009; Monden \& Hamada, 1991). 
El proceso de TC está compuesto de un número de actividades y decisiones que comienza con el diseño del producto, la definición de características, calidades y el precio ideal. El primer paso debe incluir la identificación de las ventajas competitivas, la definición de los segmentos objetivos, las simulaciones de mercado y el posicionamiento del nuevo producto en el mercado (Kocakülâh \& Austill, 2006; Sani \& Allahverdizadeh, 2012). El segundo paso en el proceso es la determinación de los deseos de beneficio o ganancia marginal; debe ser razonable y suficiente para realizar investigación y desarrollo del producto. A partir del pronóstico de la demanda y el presupuesto, se calcula el costo objetivo por unidad de producto desarrollado (Filomena, Neto \& Duffey, 2009; Sani \& Allahverdizadeh, 2012).

$$
\text { El cálculo del precio y el coste permitido }
$$
del producto es el tercer paso. Es la diferencia entre el precio objetivo y la ganancia marginal y/u objetivo. En este paso, se debe considerar lo concerniente al diseño y especificaciones del producto, realizar un sondeo del actual costo del producto apoyado en el costeo por actividades (ABC) (Sani \& Allahverdizadeh, 2012).

En el cuarto paso se determinan la naturaleza y la cantidad de producto manufacturado y sus costos de comercialización; este paso termina cuando la empresa encuentra la forma de satisfacer las necesidades de los clientes o cuando decide retirar el producto del mercado. Finalmente, el sistema debe ser implementado, evaluado y vigilado (Kocakülâh \& Austill, 2006; Sani \& Allahverdizadeh, 2012 ). En la tabla 4 aparecen algunas aplicaciones de esta metodología.

\begin{tabular}{ll}
\hline \multicolumn{1}{c}{$\begin{array}{c}\text { Sector de la } \\
\text { aplicación }\end{array}$} & \multicolumn{1}{c}{ Artículo } \\
\hline Industria automovilística & $\begin{array}{l}\text { Norhafiza Baharudin y Ruzita Jusoh (2015), Tiago Pascoal Filomena, Francisco Jos } \\
\text { Kliemann Neto \& Michael Robert Duffey (2009), Ugo Ibusuki \& Paulo Carlos Kaminski } \\
\text { (2007), Yasuhiro Monden \& Kasuki Hamada (1991) }\end{array}$ \\
Industria manufacturera & Armando Arioti, Claudio Fantozzi, Massimo Granchi \& Enrico Vettori (1999) \\
$\begin{array}{l}\text { Industria agroalimentaria } \\
\text { Operadores logísticos } \\
\text { (outsourcing) }\end{array}$ & Mehmet C. Kocakülâh \& A. David Austill (2006) \\
\hline
\end{tabular}

Tabla 4

Aplicación del target costing

Fuente: elaboración propia con base en la revisión de la literatura

\subsection{Costeo Kaizen (KC, Kaizen Costing)}

La herramienta de ingeniería de valor ha sido usada en la contabilidad por los japoneses en paralelo con TC. Mientras el TC está enfocado en el diseño del producto, el costeo Kaizen (KC,
Kaizen cost) se dirige a la fase de producción. El TC origina en la etapa de diseño una meta a cumplir por ingenieros y equipos de producción de la empresa y se convierte en un problema de la filosofía de manufactura esbelta o 
Kaizen (Amirkulova, 2011). Por su parte, el KC, denominado también costeo esbelto, es un sistema de apoyo para la reducción de costos en la fase de manufactura con una actividad de mejoramiento continuo (Monden \& Hamada, 1991). El KC se convierte en una cultura organizacional de aprendizaje colaborativo entre todos los niveles de la compañía, en especial aquellos que intervienen en los procesos de manufactura (Sani \& Allahverdizadeh, 2012).

KC es una herramienta de reducción de costo, poco a poco, e innova en cada una de las etapas propuestas en el TC, lo que hace que el KC y el TC sean inseparables (Feil, Yook \& Kim, 2004). El KC es un instrumento para cumplir metas de ganancias a corto plazo, mientras el proceso de TC se centra en metas a largo plazo, ambos manejan un enfoque de control (Feil, Yook \& Kim, 2004).

El método de mejoramiento del KC clasifica las actividades del proceso en dos tipos. El primer grupo de actividades de rendimiento real, cuando la diferencia entre el costo real y objetivo es alta en los productos de manufactura. El segundo grupo de actividades son para disminuir la diferencia entre el costo objetivo y el costo estimado de producción (Monden \& Hamada, 1991).

\subsection{Supply Chain Operations Reference (SCOR)}

El SCOR, que se define como un modelo operacional para la CS, se considera un estándar enfocado en la administración de la CS y sirve como instrumento de diagnóstico, que integra principios de reingeniería, benchmarking y ele- mentos de medición del desempeño de los procesos de la cadena.

El modelo SCOR (Supply Chain Operations Reference, modelo de referencia de operaciones de la cadena de suministro), desarrollado en 1996 por el Consejo de la CS o Supply-Chain Council (SCC), es una herramienta de gestión estratégica para tener una visión global de toda la CS. El SCOR especifica cada uno de los procesos y elementos; analiza, mide y establece objetivos de rendimiento; determina oportunidades de mejora; identifica las mejores prácticas y prioriza proyectos de mejoramiento para garantizar el cumplimiento de servicio de la red de distribución (Frazelle, 2002).

El modelo SCOR proporciona un marco único que une los procesos de negocio, los indicadores de gestión, las mejores prácticas y las tecnologías en una estructura unificada para apoyar la comunicación entre los socios de la CS, mejora las relaciones entre actividades y la eficacia de la gestión de la CS (GCS); además, permite a las organizaciones diseñar e implementar planes de mejoramiento en el corto, mediano y largo plazo. El modelo abarca las interacciones con los clientes, las transacciones físicas de materiales y todas las interacciones con el mercado (Calderón-Lama \& Lario-Esteban, 2005).

El modelo SCOR es una alternativa para conceptualizar, evaluar, balancear y mejorar sistemas logísticos, que proporciona indicadores claves de rendimiento (KPI, key performance indicators), en cinco atributos de rendimiento: fiabilidad en cumplimiento (Reliability), velocidad de atención (Responsiveness), flexibilidad (Agility), costos (Costs) y gestión de activos (As- 
costos logísticos y Metodologías PARA el COSTEO EN CADENAS / J. ORJUELA, N. SUÁREZ, y. CHINCHILLA / 395

sets Management) (Salazar-Sanabria \& LópezBello, 2009).

Aunque la clasificación de los costos no es la misma, ni las métricas iguales en cada nivel, la idea de SCOR es incluir varios niveles de agregación. Los costos en el modelo se van acumulando de proceso en proceso, a medida que se va agregando valor al producto. Clasifica los costos en manufactura, transporte, inventario, transacción, capital y de los enlaces en la cadena (Parra-Ortega, 2010).

\section{Resultados y discusión}

A continuación, la tabla 5 muestra la relación de 50 artículos de los 135 utilizados en esta revisión (gráfica 2), solo estos 50 artículos relacionan las metodologías actuales de manejo de costos, costos logísticos en CS y la relación con el desempeño de la cadena, en concordancia con la perspectiva de análisis establecida en este artículo. 
396 / VOL. 17 / NO. 44 / JULIO-DICIEMBRE 2016

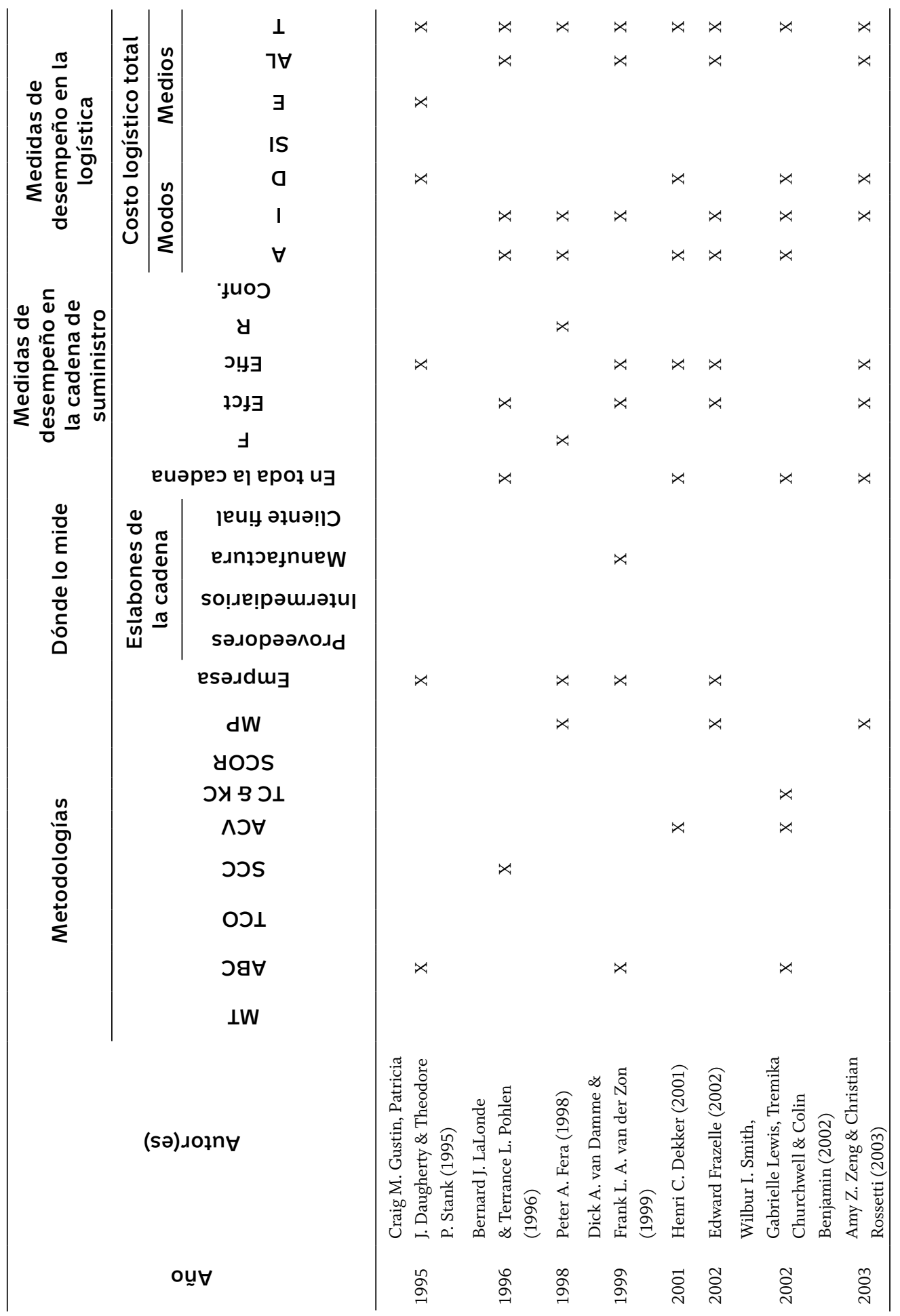


costos logísticos y Metodologías PARA el COSTEO EN CADENAS / J. ORJUELA, N. SUÁREZ, y. CHINCHILLA / 397

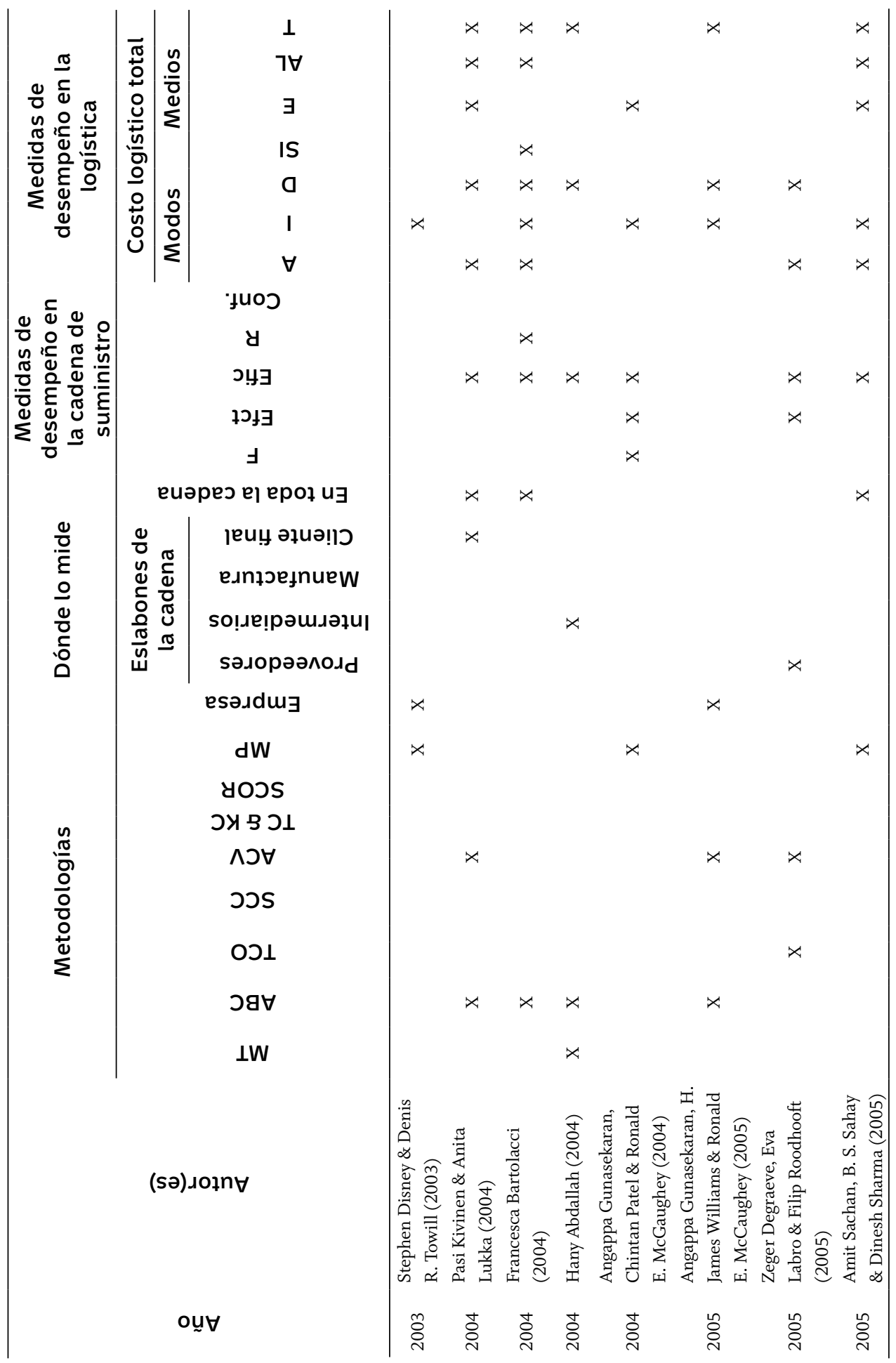




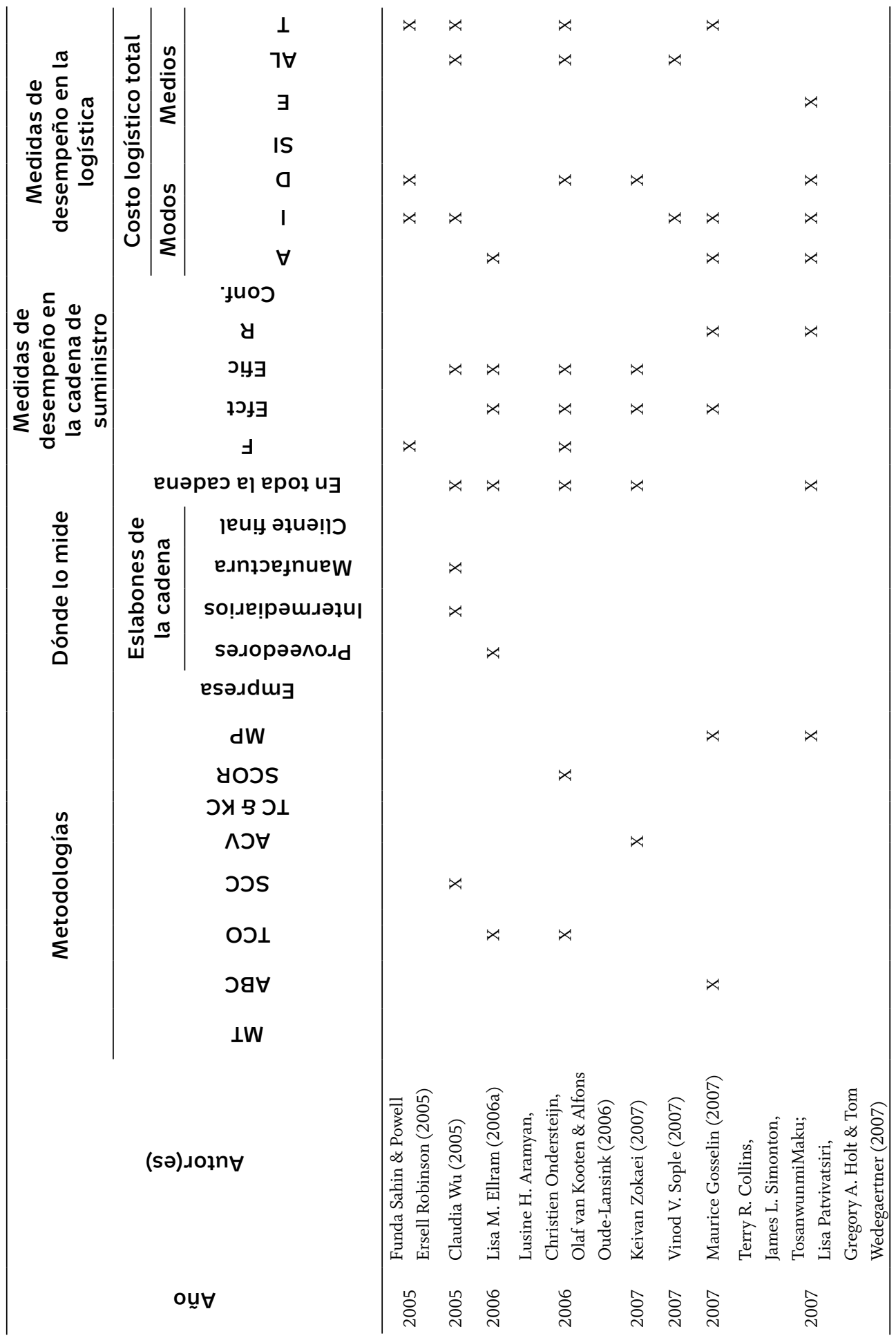


Costos Locísticos Y METOdolocías PARA EL COSTEO EN CADENAS / J. ORJUELA, N. SUÁREZ, Y. CHINCHILLA / 399

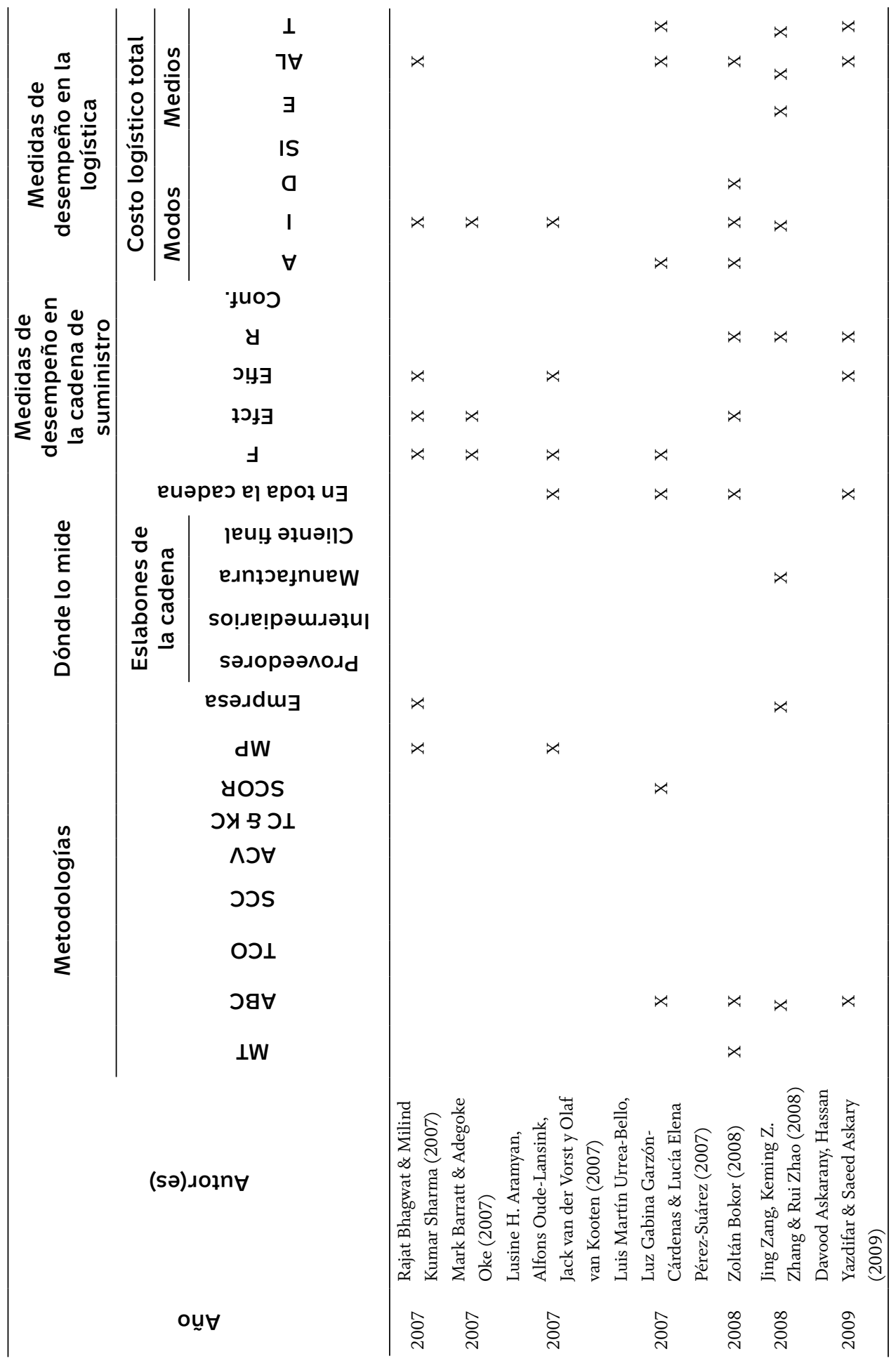


400 / VOL. 17 / NO. 44 / JULIO-DICIEMBRE 2016

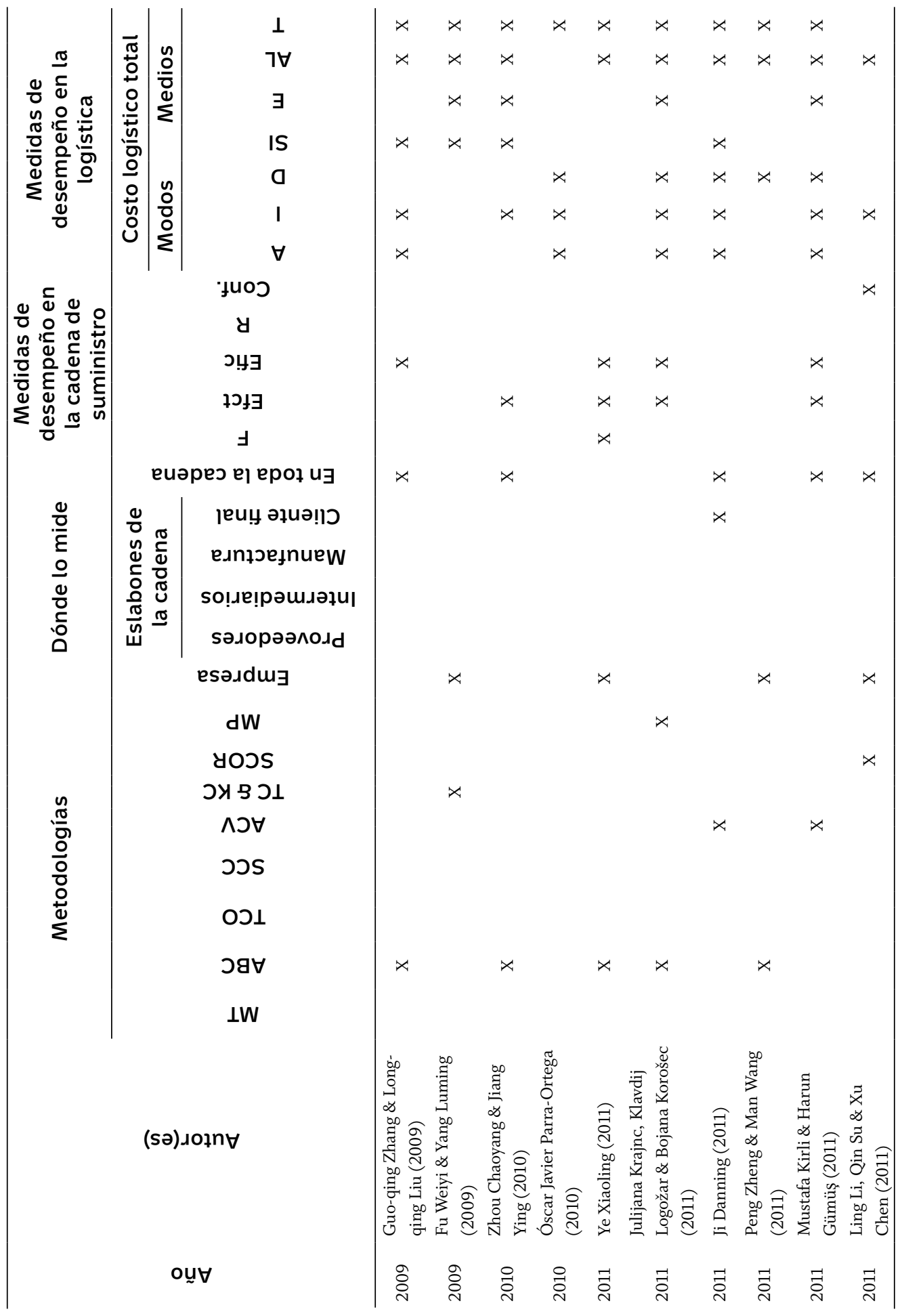


costos locísticos y Metodolocías PARA el costeo en CADENAS / J. ORJUELA, N. SUÁRez, y. CHINCHILla / 401

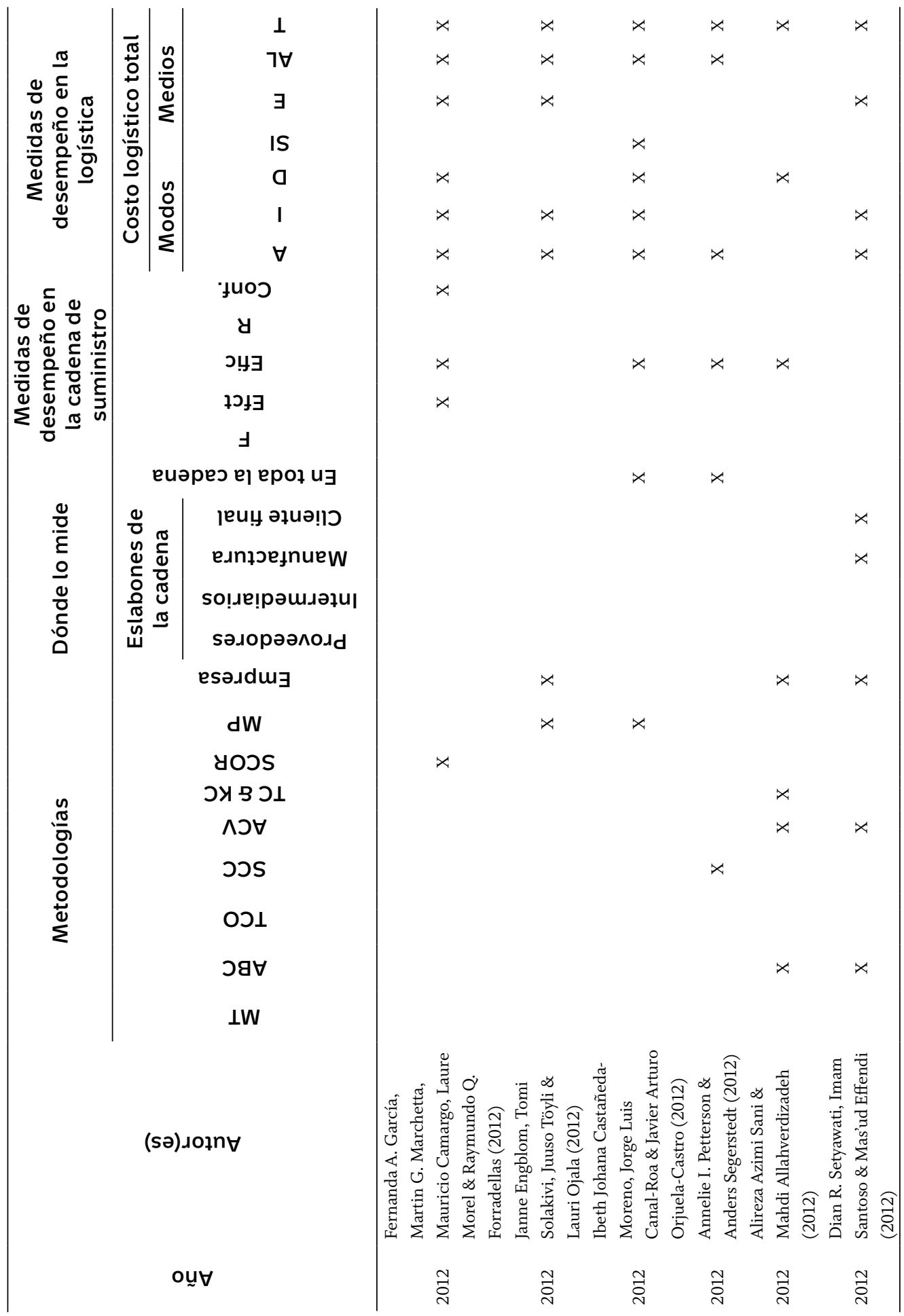




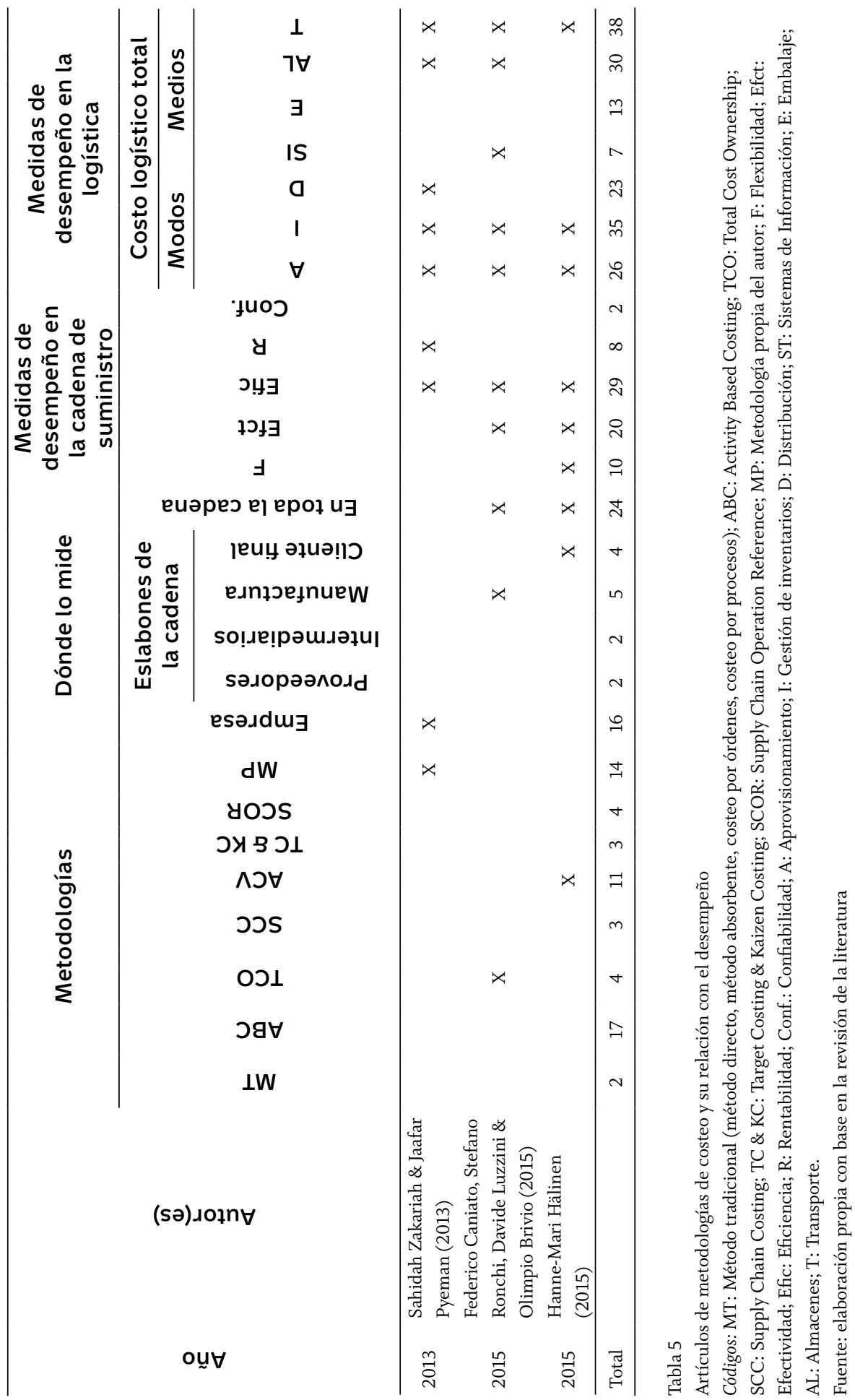


Las metodologías actuales para la medición del costo en CS se utilizan de manera aislada, es decir, se adopta una filosofía de manejo del costo total de la cadena. En algunos casos, se podrían combinar varias metodologías para mejorar los resultados, el costeo por actividades se podría integrar fácilmente el AV, el TC o el TCO como lo hacen diferentes autores y se muestra en la gráfica 6. Presenta la frecuencia de utilización de las diferentes metodologías. La de mayor frecuencia de uso en las CS es el costeo basado en actividades (36\%), seguido de las metodologías propias de diferentes autores (28\%), luego las metodologías de AV (11\%), TCO (8\%), TC, SCOR, SCC con un $6 \%$, y el método tradicional de costeo en último lugar en aplicaciones en costeo logístico.
A partir de los datos anteriores, se evaluó si las metodologías que contemplaban la totalidad de la cadena o algunos eslabones de la cadena gráfica 7 . Muestra la frecuencia con que se utilizan las metodologías, la totalidad de la cadena es el tópico más recurrente para aplicar y analizar los costos logísticos y su relación con el desempeño (48\%), seguido de la empresa para mejorar la eficiencia (32\%), metodologías como la utilizada por el AV se enfoca en el cliente final (6\%), en los demás eslabones las metodologías se aplican en 4\%.

La mayor parte de los autores utilizan metodologías para costear logística en la cadena e incluyen medidas de desempeño de la CS. La gráfica 8 presenta algunas de las medidas, la eficiencia de la CS con 58\% es la más empleada para la logística, la efectividad (40\%), flexibilidad (22\%), rentabilidad (16\%), confiabilidad (4\%).

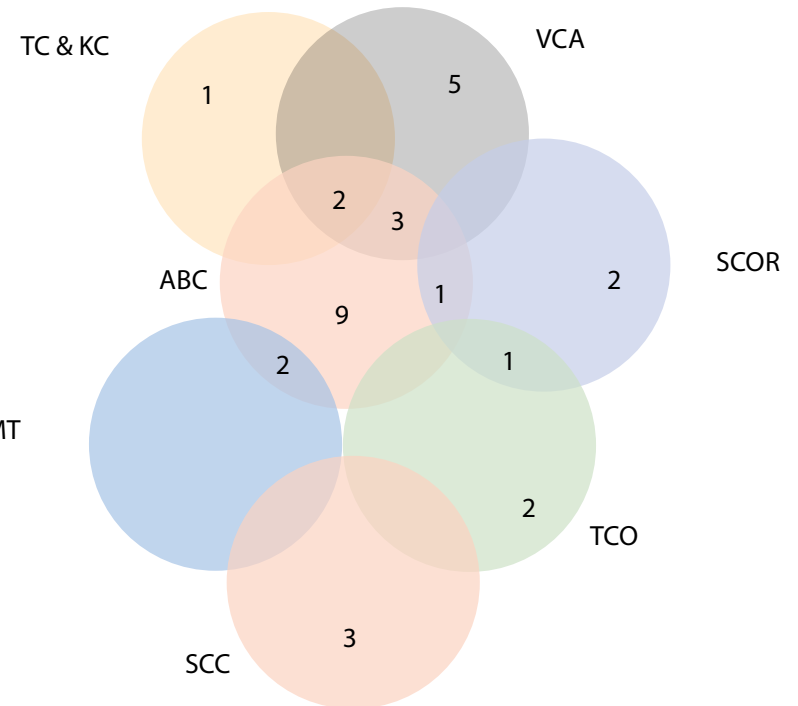

\section{Gráfica 6}

Frecuencias de uso de las metodologías Fuente: elaboración propia 


\section{4 / VOL. 17 / NO. 44 / JULIO-DICIEMBRE 2016}

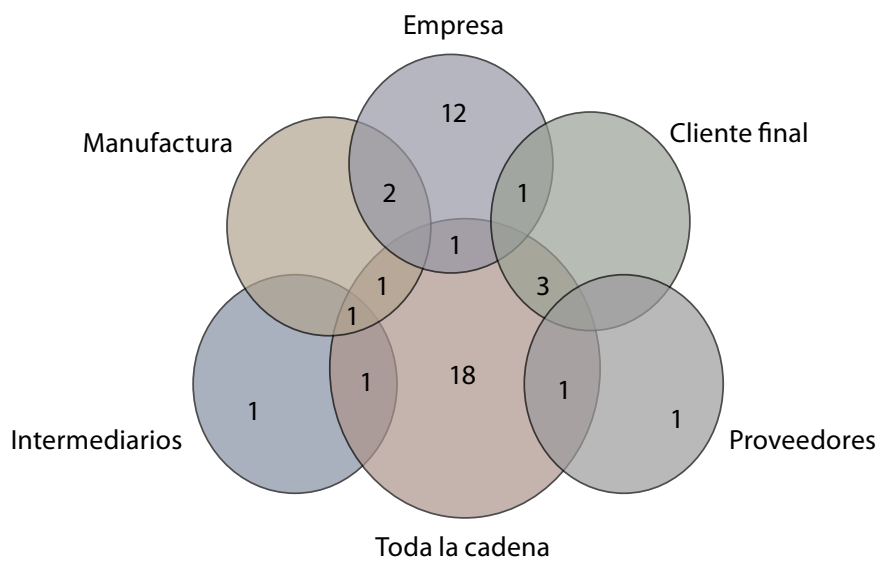

Gráfica 7

Frecuencia de uso de las metodologías en los eslabones de la cadena de suministro

Fuente: elaboración propia

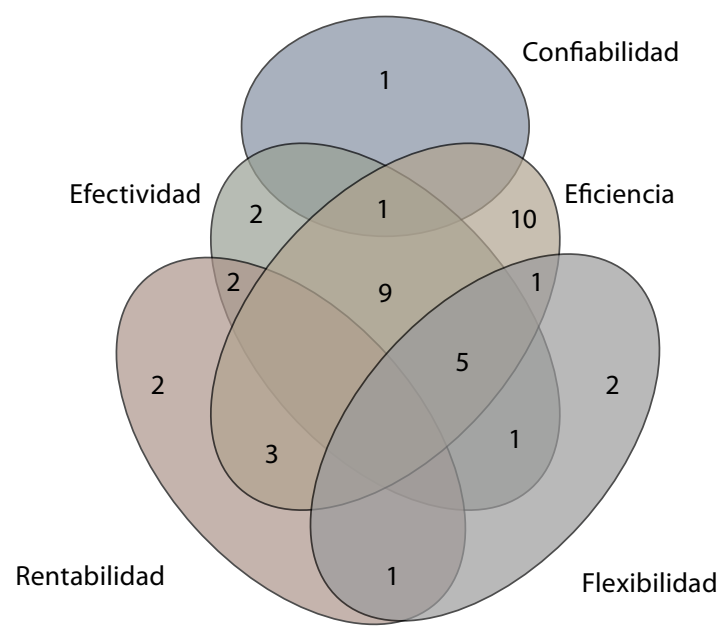

\section{Gráfica 8}

Frecuencia de uso de medidas de desempeño en cadenas de suministro

Fuente: elaboración propia

Frente a la medición del desempeño logístico con base en el costo logístico total, la gráfica 9 muestra la cantidad de publicaciones que utilizan en la metodología los costos clasificados en medios y modos. 
MEDIOS (Costo de aprovisionamiento, Costo de inventarios, Costo de distribución)

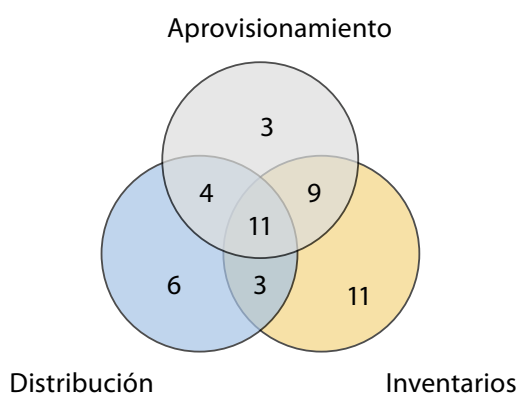

MODOS (Costo de Sistemas de Información, Costo de transporte, Costo de almacenes)

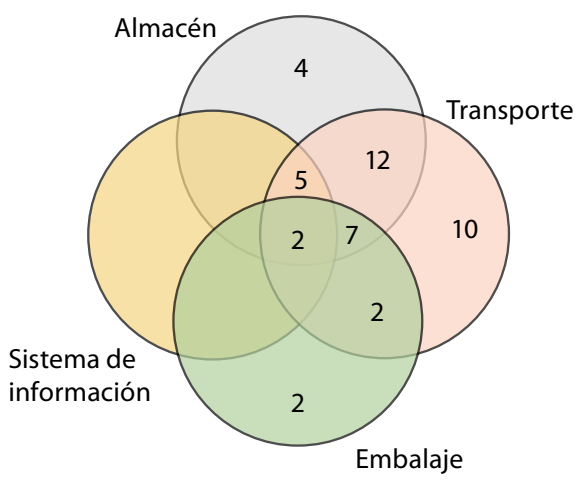

\section{Gráfica 9}

Frecuencia de ocurrencia en medios y modos Fuente: elaboración propia

Los valores de la gráfica 9 representan las veces que estos costos fueron incluidos en cualquier metodología expuesta. 38 de las 50 publicaciones incluyeron costo de transporte (76\%); 35, el costo de inventario (70\%); 30, el costo de almacenamiento o gestión de almacén (60\%); 26, el costo de aprovisionamiento (52\%); 23 incluyeron el costo de distribuir (46\%); 13, el costo de embalaje (26\%) y 7 artículos involucraron el costo de la información (14\%).

A pesar de que las metodologías estudiadas tienen en cuenta los costos de la CS completa, muy pocas están destinadas a costear únicamente el área logística considerando los cinco procesos principales, lo cual resalta la necesidad de encontrar una metodología que esté enfocada en identificar y medir el costo total de la logística.

Después de haber abordado las metodologías más relevantes para el costeo en CS, la tabla 6 presenta los costos que los autores han referido de cada una, con el fin de darle al lector una visión comparativa de cada metodología al enfrentarse a un problema de costeo.

La tabla 7 compara las diferentes perspectivas de los autores, frente a los procesos. Esto se presenta dado que es posible que cada metodología, además de clasificar las categorías de costo de manera diferente, también analice los procesos desarrollados en la empresa, a través de una clasificación específica de procesos. 


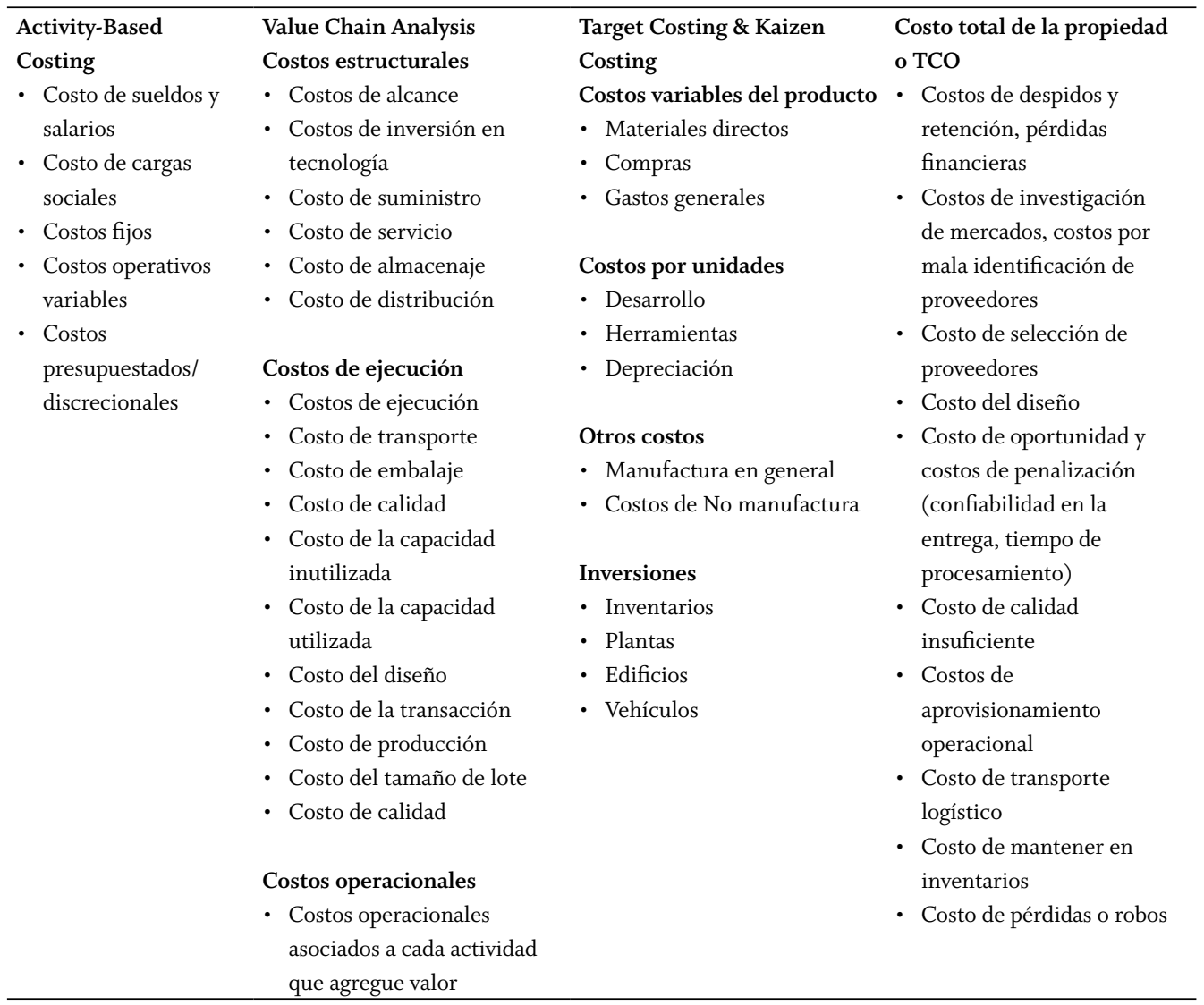

Tabla 6

Costos incluidos en cada una de las metodologías Fuente: elaboración propia

\begin{tabular}{|c|c|c|c|}
\hline $\begin{array}{c}\text { Grupo de investigación } \\
\text { GICALYT }\end{array}$ & Value Chain Analysis & SCOR & $\begin{array}{l}\text { Supply Chain } \\
\text { Costing }\end{array}$ \\
\hline $\begin{array}{l}\text { - Aprovisionamiento } \\
\text { - Almacenamiento } \\
\text { - Inventario } \\
\text { - Distribución } \\
\text { - Logística de servicio al cliente }\end{array}$ & $\begin{array}{l}\text { - Logística de entrada (compras, } \\
\text { aprovisionamiento, transporte } \\
\text { interno, almacenamiento) } \\
\text { - Operaciones internas } \\
\text { (manufactura, producción, } \\
\text { desperdicios) } \\
\text { - Logística de salida (servicio } \\
\text { al cliente, almacenamiento, } \\
\text { transporte) } \\
\text { - Distribución } \\
\text { - Comercialización y marketing }\end{array}$ & $\begin{array}{l}\text { - Planeación } \\
\text { - Fuente } \\
\text { (almacenamiento, } \\
\text { compra, } \\
\text { inventario) } \\
\text { - Producción o } \\
\text { manufactura } \\
\text { - Distribución } \\
\text { - Retorno }\end{array}$ & $\begin{array}{l}\text { - Aprovisionamiento } \\
\text { - Manufactura } \\
\text { - Almacenamiento e } \\
\text { inventarios } \\
\text { - Distribución } \\
\text { - Servicio al cliente }\end{array}$ \\
\hline
\end{tabular}

Tabla 7

Clasificación de los procesos de acuerdo a la metodología utilizada

Fuente: elaboración propia 
La tabla 8, a manera de síntesis, muestra las diferencias entre las metodologías analizadas previamente, en la cual se incluyen la forma de analizar los costos, el objetivo, los inductores, la filosofía de costeo y la naturaleza de la información.

\begin{tabular}{|c|c|c|c|c|}
\hline & $\begin{array}{c}\text { Sistemas } \\
\text { tradicionales de } \\
\text { contabilidad }\end{array}$ & $\begin{array}{c}\text { Costeo Basado } \\
\text { en Actividades } \\
(A B C)\end{array}$ & $\begin{array}{l}\text { Costeo de la } \\
\text { Cadena de Valor } \\
\text { (Value Chain } \\
\text { Costing) }\end{array}$ & $\begin{array}{c}\text { Costeo por } \\
\text { Objetivos y } \\
\text { Mejoramiento } \\
\text { continuo (Target } \\
\text { Costing \& Kaizen } \\
\text { Costing) }\end{array}$ \\
\hline Enfoque & Interno & Interno & Externo & Interno/Externo \\
\hline $\begin{array}{l}\text { Forma de } \\
\text { analizar los } \\
\text { costos }\end{array}$ & $\begin{array}{l}\text { En términos del } \\
\text { producto y del cliente, } \\
\text { con un enfoque } \\
\text { interno arraigado. } \\
\text { Agregar valor es un } \\
\text { concepto clave. }\end{array}$ & $\begin{array}{l}\text { Asigna costos directos e } \\
\text { indirectos a actividades } \\
\text { que consumen } \\
\text { recursos. Luego, } \\
\text { combina los costos } \\
\text { en relación con los } \\
\text { recursos usados. }\end{array}$ & $\begin{array}{l}\text { En términos de varias } \\
\text { etapas de la cadena } \\
\text { de valor o los que la } \\
\text { empresa defina. Tiene } \\
\text { un enfoque externo } \\
\text { determinante. }\end{array}$ & $\begin{array}{l}\text { Se define en tres etapas } \\
\text { definitivas del proceso: } \\
\text { diseño, desarrollo y } \\
\text { producción. Tiene } \\
\text { un enfoque externo e } \\
\text { interno en el que se } \\
\text { combinan investigaciones } \\
\text { internas y externas. }\end{array}$ \\
\hline $\begin{array}{l}\text { Objetivo del } \\
\text { análisis de } \\
\text { costos }\end{array}$ & $\begin{array}{l}\text { Tiene tres objetivos } \\
\text { clave en el desarrollo } \\
\text { de este método: } \\
\text { Detectar problemas } \\
\text { Dirigir la atención } \\
\text { Solucionar problemas }\end{array}$ & $\begin{array}{l}\text { Lograr una mayor } \\
\text { eficiencia en los } \\
\text { procesos. Inicia con } \\
\text { la identificación } \\
\text { y definición de } \\
\text { actividades que } \\
\text { componen los procesos } \\
\text { y mide el consumo de } \\
\text { recursos. }\end{array}$ & $\begin{array}{l}\text { Maneja los mismos tres } \\
\text { objetivos, pero utiliza } \\
\text { estrategias diferentes } \\
\text { para alcanzarlos } \\
\text { como estrategias de } \\
\text { liderazgo o estrategias } \\
\text { de diferenciación de la } \\
\text { marca. }\end{array}$ & $\begin{array}{l}\text { Los objetivos se basan en } \\
\text { actividades para alcanzar } \\
\text { el costo objetivo antes de } \\
\text { una producción en masa } \\
\text { con esfuerzos continuos } \\
\text { e innovación. }\end{array}$ \\
\hline $\begin{array}{l}\text { Concepto de los } \\
\text { inductores de } \\
\text { costo }\end{array}$ & $\begin{array}{l}\text { Tiene un solo inductor } \\
\text { de costo que se utiliza } \\
\text { para alcanzar los } \\
\text { objetivos. }\end{array}$ & $\begin{array}{l}\text { Los inductores de costo } \\
\text { se emplean para reflejar } \\
\text { el consumo de costos } \\
\text { por las actividades y, a } \\
\text { su vez, el consumo de } \\
\text { las actividades por los } \\
\text { productos. }\end{array}$ & $\begin{array}{l}\text { Maneja tres inductores } \\
\text { de costo: estructurales, } \\
\text { de ejecución, } \\
\text { operacionales. } \\
\text { Cada actividad tiene una } \\
\text { parte del inductor de } \\
\text { costo. }\end{array}$ & $\begin{array}{l}\text { Los inductores de } \\
\text { costo se dirigen al } \\
\text { cumplimiento del precio } \\
\text { objetivo, costo objetivo, } \\
\text { ganancia marginal, costo } \\
\text { del mejoramiento. }\end{array}$ \\
\hline $\begin{array}{l}\text { Filosofía del } \\
\text { costeo }\end{array}$ & $\begin{array}{l}\text { Identificar y } \\
\text { reducir el costo con } \\
\text { responsabilidades } \\
\text { y cuestiones del } \\
\text { producto }\end{array}$ & $\begin{array}{l}\text { El cálculo de costos } \\
\text { basado en actividades } \\
\text { se fundamenta en la } \\
\text { premisa de que los } \\
\text { productos requieren } \\
\text { que una empresa } \\
\text { ejecute determinadas } \\
\text { actividades y que tales } \\
\text { actividades requieren } \\
\text { que se incurra en } \\
\text { costos. }\end{array}$ & $\begin{array}{l}\text { Identificar y reducir } \\
\text { el costo al regular los } \\
\text { inductores de costo en } \\
\text { cada actividad. }\end{array}$ & $\begin{array}{l}\text { Reduce el costo mediante } \\
\text { el conocimiento del } \\
\text { diseño del producto } \\
\text { manteniendo políticas de } \\
\text { mejoramiento continuo e } \\
\text { independiente. }\end{array}$ \\
\hline
\end{tabular}




\begin{tabular}{|c|c|c|c|c|}
\hline & $\begin{array}{c}\text { Sistemas } \\
\text { tradicionales de } \\
\text { contabilidad }\end{array}$ & $\begin{array}{c}\text { Costeo Basado } \\
\text { en Actividades } \\
(A B C)\end{array}$ & $\begin{array}{l}\text { Costeo de la } \\
\text { Cadena de Valor } \\
\text { (Value Chain } \\
\text { Costing) }\end{array}$ & $\begin{array}{c}\text { Costeo por } \\
\text { Objetivos y } \\
\text { Mejoramiento } \\
\text { continuo (Target } \\
\text { Costing \& Kaizen } \\
\text { Costing) }\end{array}$ \\
\hline Enfoque & Interno & Interno & Externo & Interno/Externo \\
\hline $\begin{array}{l}\text { Aplicación en } \\
\text { administración }\end{array}$ & $\begin{array}{l}\text { Planeación de } \\
\text { presupuestos } \\
\text { Control de costos } \\
\text { Simplificación de } \\
\text { actividades }\end{array}$ & $\begin{array}{l}\text { Toma de decisiones, } \\
\text { subcontratación y } \\
\text { análisis de rentabilidad. }\end{array}$ & $\begin{array}{l}\text { Costos y actividades } \\
\text { que afectan el valor del } \\
\text { producto } \\
\text { Reconfiguración de la } \\
\text { cadena de suministro y } \\
\text { explotación de vínculos }\end{array}$ & $\begin{array}{l}\text { Reducción de costos } \\
\text { Ajuste a las presiones } \\
\text { sobre las actividades } \\
\text { Reduce costo sobre los } \\
\text { productos en proceso }\end{array}$ \\
\hline $\begin{array}{l}\text { Objetivo } \\
\text { primordial }\end{array}$ & $\begin{array}{l}\text { Impacto del costo en la } \\
\text { utilidad. }\end{array}$ & $\begin{array}{l}\text { Identificación de } \\
\text { costos. }\end{array}$ & $\begin{array}{l}\text { Costo, valor y enlaces en } \\
\text { la cadena. }\end{array}$ & $\begin{array}{l}\text { Disminución e impacto } \\
\text { de los costos en la } \\
\text { utilidad. }\end{array}$ \\
\hline $\begin{array}{l}\text { Naturaleza de la } \\
\text { información }\end{array}$ & Información interna. & Información interna. & $\begin{array}{l}\text { Información interna y } \\
\text { externa. }\end{array}$ & $\begin{array}{l}\text { Información interna y } \\
\text { externa. }\end{array}$ \\
\hline
\end{tabular}

Tabla 8

Aspectos diferenciadores de las metodologías actuales de costeo

Fuente: elaboración propia a partir de Francesca Bartolacci (2004), Federico Caniato, Stefano Ronchi, Davide Luzzini y Olimpio Brivio (2015), Joseph L. Cavinato (1992), Douglas T. Hicks (1998), Mustafa Kirli y Harun Gümüş (2011), María Rosa López-Mejía, Alicia Gómez-Martínez \& Salvador Marín-Hernández (2011), Maria da Conceição da Costa Marques (2012), Yasuhiro Monden \& Kasuki Hamada (1991), Terrance L. Pohlen \& Bernard J. LaLonde (1994), Michael Porter (1990), Alireza Azimi Sani y Mahdi Allahverdizadeh (2012), John K. Shank y Vijay Govindarajan (1993).

\section{Conclusiones}

Los costos logísticos en la CS no son un concepto nuevo en la literatura, su evolución ha sido constante y paulatina con el paso del tiempo, impulsado por la competitividad como directriz y guía para el éxito de los negocios. Los costos logísticos y su relación con el desempeño de la CS han sido ampliamente estudiados; no obstante, falta concretar una metodología que pueda determinar claramente cómo costear la logística de las empresas únicamente.

La metodología de costeo por actividades parece la más adecuada para obtener una aproximación al costo logístico total; sin embargo, esta revisión ha determinado que muy pocos autores han destinado su trabajo a la aplicación de una o más metodologías para el costo de los procesos logísticos. En su mayoría, las publicaciones han optado por costeo por actividades, aunque el análisis de cadena de valor está en auge, en concordancia con la atención de los actores en la creación de ventajas competitivas y la opinión del cliente para el ajuste de sus precios, siempre manteniendo la premisa de entregar productos de calidad a un costo menor.

Se ha presentado un marco de análisis de las metodologías de costeo para la CS, a partir de la cual se ha propuesto una taxonomía sobre medidas de desempeño para la cadena y la logística de la cadena, en cada eslabón o en la empresa. 


\section{Referencias}

Abdallah, Hany (2004). Guidelines for Assessing Cost in a Logistics System: An Example of Transport Cost Analysis. Arlington, Virginia: John Snow, Inc./Deliver. Disponible en: http://www.jsi.com/ JSIInternet/Inc/Common/_download_pub. cfm?id=11102\&lid=3

Adams, Mike (1996). Activity-Based Costing and the Life-Insurance Company. Service Industries Journal, 16 (4), 511-526.

Aird, Brian (1996). Activity-Based Cost Management in Health Care - Another Fad? International Journal of Health Care Quality Assurance, 9 (4), 16-19.

Amirkulova, Maral B. (2011). Expenses Management Tools at the Enterprises of the Republic of Kazakstan. Education and Science without Borders, 2 (4), 7-9.

Anderson, Terry \& Elloumi, Fathi (2004). Theory and Practice of Online Learning. Disponible en: http://cde.athabascau.ca/ online_book/pdf/TPOL_book.pdf

Aramyan, Lusine H.; Ondersteijn, Christien; Kooten, Olaf van \& Oude-Lansink, Alfons (2006). Performance Indicators in Agri Food Production Chains. Quantifying the Agri-Food Supply Chain, 15, 47-64. Disponible en: http://library. wur.nl/WebQuery/wurpubs/list?wq_ inf1=p1030072\&A114=1030072

Aramyan, Lusine H.; Oude-Lansink, Alfons; Vorst, Jack van der \& Kooten, Olaf van (2007). Performance Measurement in Agri-Food Supply Chains: A Case Study. Supply Chain Management an International
Journal, 12, 304-315. Disponible en: http:// library.wur.nl/WebQuery/wurpubs/list?wq inf1 $=$ p1030072\&A114=1030072

Arioti, Armando; Fantozzi, Claudio; Granchi, Massimo \& Vettori, Enrico (1999). A Methodology to Diagnose the Target Cost in a Manufacturing Process. Proceedings of the Second International Conference on Intelligent Processing and Manufacturing of Materials, 1999, IPMM ‘99, 1, 629-633.

Askarany, Davood; Yazdifar, Hassan \& Askary, Saeed (2009). Supply Chain Management, Activity-Based Costing and Organisational Factors. International Journal Production Economics, 127, 238-248. Disponible en: http:// papers.ssrn.com/sol3/papers.cfm?abstract_ id $=1370783$

Baharudin, Norhafiza \& Jusoh, Ruzita (2015). Target Cost Management (TCM): A Case Study an Automotive Company. Procedia - Social and Behavioral Sciences, 172 (27), 525-532. Disponible en: http://www.sciencedirect.com/science/ journal/18770428/172/supp/C

Ballou, Ronald H. (2004). Logística: administración de la cadena de suministro. Juárez: Pearson Educación. Disponible en: http:// datateca.unad.edu.co/contenidos/102508/ Entorno_e_conocimiento_2016/ Logistica_Administracion_de_la_ cadena_de_suministro_5ta_Edicion_-Ronald_H._Ballou.pdf

Barnes, Stuart J. (2002). The Mobile Commerce Value Chain: Analysis and Future Developments. International Journal of Information Management, 22 (2), 91-108. Disponible en: https:// 
pdfs.semanticscholar.org/0b29/

c355309fc0ee405dbadafa9bacf683e89910.

pdf

Barratt, Mark \& Oke, Adegoke (2007).

Antecedents of Supply Chain Visibility

in Retail Supply Chains: A Resource-

Based Theory Perspective. Journal of

Operations Management, 25 (6), 1217-1233.

Disponible en: https://www.researchgate. net/publication/216020391_Antecedents_ of_Supply_Chain_Visibility_in_Retail_ Supply_Chains_A_Resource-Based_ Theory_Perspective

Bartolacci, Francesca (2004). Activity Based Costing in the Supply Chain. Logistics Activities Cost Analysis. Temi di discussione, 23, Università degli Studi di Macerata, Dipartimento di Istituzioni Economiche e Finanziarie. Disponible en: http://citeseerx.ist. psu.edu/viewdoc/download?doi=10.1.1.524. $8387 \&$ rep $=$ rep $1 \&$ type $=$ pdf

Bhagwat, Rajat \& Sharma, Milind Kumar (2007). Performance Measurement of Supply Chain Management: A Balanced Scorecard Approach. Computers \& Industrial Engineering, 53, 43-62. Disponible en: http://www.academia.edu/1140263/ Performance_measurement_of_supply_ chain_management_A_balanced_ scorecard_approach

Bjørnenak, Trond (2000). Understanding Cost Differences in the Public Sector - A Cost Drivers Approach. Management Accounting Research, 11 (2), 193-211. Disponible en: http://isiarticles.com/bundles/Article/pre/ pdf/8184.pdf
Bokor, Zoltán (2008). Supporting Logistics Decisions by Using Cost and Performance Management Tools. Transportation Engineering, 36 (1-2), 33-39. Disponible en: https:// pp.bme.hu/tr/2008_1/pdf/tr2008_1_07.pdf

Bowersox, Donald J.; Closs, David J. \& Cooper, Bixby (2007). Supply Chain Logistics Management. New York: McGraw-Hill.

Bremen, Philipp; Oehmen, Josef \& Alard, Robert (2007). Cost-Transparent Sourcing in China Applying Total Cost of Ownership. Proceedings of the 2007 IEEE IEEM, $13^{\text {th }}$ IEEE International Conference on Industrial Engineering and Engineering Management, IEEE, 262-266.

Bustamante-Salazar, Alina Marcela (2014). Costeo Basado en Actividades: Revisión de la Literatura. Revista CEA, 1 (1), 109-119. Disponible en: http://itmojs.itm.edu.co/ index.php/revista-cea/article/view/706/693 Calderón-Lama, José Luis \& Lario-Esteban, Francisco Cruz (2005). Análisis del modelo SCOR [Supply Chain Operations Reference] para la gestión de la cadena de suministro. IX Congreso de Ingeniería de Organización, Gijón, 8 y 9 de septiembre de 2005. Disponible en: http://recetasdepostres.mex.tl/ images/31616/modeloscor.pdf

Caniato, Federico; Ronchi, Stefano; Luzzini, Davide \& Brivio, Olimpio (2015). Total Cost of Ownership along the Supply Chain: A Model Applied to the Tinting Industry. Production Planning \& Control: The Management of Operations, 25 (6), 427-437.

Castañeda-Moreno, Ibeth Johana; Canal-Roa, Jorge Luis \& Orjuela-Castro, Javier Arturo (2012). Caracterización de la logística de la 
cadena de abastecimiento agroindustrial frutícola en Colombia. Bogotá: Universidad Distrital Francisco José de Caldas.

Castellani, Stefania; Grasso, Antonietta; O'Neill, Jacki \& Tolmie, Peter (2005). Total Cost of Ownership: Issues around Reducing Cost of Support in a Manufacturing Organization Case. Xerox Research Centre Europe. Disponible en: http:// xrce.xerox.com/layout/set/print/ content/download/18781/134103/file/ BusinessAgility.PDF

Cavinato, Joseph L. (1992). A Total Cost/Value Model for Supply Chain Competitiveness. Journal of Business Logistics, 13 (2), 285-301. Chaoyang, Zhou \& Ying, Jiang (2010). Research on Controlling Supply Chain Logistics Cost Based on Activity-Based Costing. Proceedings of the $7^{\text {th }}$ International Conference on Innovation \& Management, 1678-1682. Disponible en: http://www.pucsp.br/icim/ ingles/downloads/papers_2010/part_7/36_ Research\%20on\%20Controlling\%20 Supply\%20Chain\%20Logistics\%20 Costs\%20Based\%20on.pdf

Christopher, Martin (2011). Logistics \& Supply Chain Management. London: Pearson Education Limited.

Cokins, Gary (2002). Integrating Target Costing and ABC. Journal of Cost Management, 16 (4), 13-22. Disponiuble en: https://www. researchgate.net/publication/292738141_ Integrating_target_costing_and_ABC Cokins, Gary (2006). Implementing ActivityBased Costing. Statements on Management Accounting, Institute of Management Accounting, 1-33. Disponible en: http://www. imanet.org/docs/default-source/research/ sma/implementing-activity-based-costing. pdf?sfvrsn=2

Collins, Terry R.; Simonton, James L.; Maku, T Tosanwunmi; Patvivatsiri, Lisa; Holt, Gregory A. \& Wedegaertner, Tom (2007). The Development of Cost Model for a New Agricultural Product. Proceedings of the Industrial Engineering Research Conference, Nashville.

Cooper, Robin \& Kaplan, Robert S. (1988). Measure Cost Right: Make the Right Decisions. Harvard Business Review, 66 (5), 96-103. Disponible en: https://hbr. org/1988/09/measure-costs-right-make-theright-decisions

Cooper, Robin \& Kaplan, Robert S. (1991). Profit Priorities from Activity-Based Costing. Harvard Business Review, 69 (3), 130-135. Disponible en: https://hbr. org/1991/05/profit-priorities-from-activitybased-costing

Cooper, Robin \& Slagmulder, Regine (1997). Target Costing and Value Engineering. Portland, Oregon: Productivity Press.

Daganzo, Carlos F. (2005). Logistics System Analysis. Berlin: Springer.

Damme, Dick A. van \& Zon, Frank L. A. van der (1999). Activity Based Costing and Decision Supporting. International Journal of Logistics Management, 10 (1), 71-83.

Danning, Ji (2011). Research on Agriculture Logistics Cost Based on Value Chain. International Conference on Information Management and Engineering, ICIME, Singapore. Disponible en: http://www.ipcsit.com/ vol52/052-ICIME2011-Contents.pdf 
Degraeve, Zeger; Labro, Eva \& Roodhooft, Filip (2000). An Evaluation of Vendor Selection Models from a Total Cost of Ownership Perspective. European Journal of Operational Research, 125 (1), 34-58.

Degraeve, Zeger; Labro, Eva \& Roodhooft, Filip (2005). Constructing a Total Cost of Ownership Supplier Selection Methodology Based on Activity Based Costing and Mathematical Programming. Accounting and Business Research, 35 (1), 3-27.

Dekker, Henri C. (2001). Accounting Information and Value Chain Analysis: An Exploratory Field Study. Amsterdam Research Center in Accounting, ARCA, Research Memorandum ARC A-RM-0 1-11, 1-41. Disponible en: ftp://ftp.repec.org/opt/ReDIF/RePEc/vua/ wpaper/pdf/2001011a.pdf

Disney, Stephen \& Towill, Denis R. (2003). The Effect of Vendor Managed Inventory (VMI) Dynamics on the Bullwhip Effect in Supply Chains. International Journal of Production Economics, 85 (2), 199-215.

Duruflé, F. (1993). Metodología general sobre el análisis de cadenas. Italia.

Eggers, John L. \& Bangert, Charles E. (1998). Activity Based Costing. Journal American Water Works Association, 90 (6), 63-60. Elloumi, Fathi (2004). Value Chain Analysis: A Strategic Approach to Online Learning. En Theory and Practice Online Learning, 6192. Athabasca, Canada: Creative Commons. Disponible en: http://cde.athabascau.ca/ online_book/

Ellram, Lisa M. (1995). Total Cost of Ownership: An Analysis Approach for Purchasing.
International Journal of Physical Distribution \& Logistics Management, 25 (8), 4-23.

Ellram, Lisa M. (2006a). TCO-Adding Value to the Supply Chain. Creating and Managing Value in Supply Networks. $15^{\text {th }}$ Annual IPSERA Conference Proceedings.

Ellram, Lisa M. (2006b). Total Cost of Ownership: Elements and Implementation. Journal of Supply Chain Management, 29 (3), 2-11.

Ellström, Daniel; Rehme, Jakob; Björklund, Maria \& Aronsson, Håkan (2012). Logistics Cost Management Models and Their Usability for Purchasing. Journal of Modern Accounting and Auditing, 8 (7), 1066-1073. Disponible en: http://www. davidpublishing.com/davidpublishing/ Upfile/9/26/2012/2012092683199265.pdf Engblom, Janne; Solakivi, Tomi; Töyli, Juuso \& Ojala, Lauri (2012). Multiple Method Analysis of Logistics Costs. International Journal of Production Economics, 137 (1), 2935. Disponible en: http://www.academia. edu/17512617/Multiple-method_analysis_ of_logistics_costs

Everaert, Patricia; Bruggeman, Werner; Sarens, Gerrit; Anderson, Steven R. \& Levant, Yves (2008). Cost Modeling in Logistics Using Time-Driven ABC: Experiences from a Wholesaler. International Journal of Physical Distribution \& Logistics Management, 38 (34), 172-191.

Donelan, Joseph G. \& Kaplan, Edward A. (1998). Value Chain Analysis: A Strategic Approach To Cost Management. Journal of Cost Management, 12 (2), 7-15. Feil, Patrick; Yook, Keun-Hyo \& Kim, IlWoon (2004). Japanese Target Costing: 
A Historical Perspective. International Journal of Strategic Cost Management, 1, 1019. Disponible en: https://www.uakron. edu/cba/docs/ins-cen/igb/scm/TCHistory_ formatted.pdf

Fera, Peter A. (1998). Developing a Framework for International Outsourcing in the Commercial Aircraft Industry. Massachusetts Institute of Technology, 1-104.

Filomena, Tiago Pascoal; Neto, Francisco Jos Kliemann \& Duffey, Michael Robert (2009). Target Costing Operationalization during Product Development: Model and Application. International Journal of Production Economics, 118 (2), 398-409.

Frazelle, Edward (2002). Supply Chain Strategy: The Logistics of Supply Chain Management. New York: McGraw-Hill Education.

Garfamy, Reza Mohammady (2006). A Data Envelopment Analysis Approach Based on Total Cost of Ownership for Supplier Selection. Journal of Enterprise Information Management, 19 (6), 662-678.

García, Fernanda A.; Marchetta, Martin G.; Camargo, Mauricio; Morel, Laure \& Forradellas, Raymundo Q. (2012). A Framework for Measuring Logistics Performance in the Wine Industry. International Journal Production Economics, 135 (1), 284-298.

Garry, Michael (1996). ABC in Action. Progressive Grocer, 75 (2), 71.

Gómez-Montoya, Luis Fernando; DuqueRoldán, María Isabel \& Carmona-López, Jamer (2009). La información contable y de costos en las entidades de salud: una herramienta para su transformación. Contaduría Universidad de Antioquia,
53, 145-172. Disponible en: revinut. udea.edu.co/index.php/cont/article/ download/2208/1779

Gosselin, Maurice (2007). A Review of ActivityBased Costing: Technique, Implementation, and Consequences. En Christopher S. Chapman, Anthony G. Hopwood \& Michael D. Shields (eds.). Handbook of Management Accounting Research, Vol. 2, 641-671. Amsterdam: Elsevier Ltd.

Groot, Tom (1999). Activity Based Costing in US and Dutch Food Companies. Advances in Management Accounting, 7, 47-63.

Gudehus, Timm \& Kotzab, Herbert (2009). Comprehensive Logistics. Berlin: Springer. Gunasekaran, Angappa; Patel, Chintan \& McGaughey, Ronald E. (2004). A Framework for Supply Chain Performance Measurement. International Journal Production Economics, 87, 333-

347. Disponible en: https://www. umassd.edu/media/umassdartmouth/ businessinnovationresearchcenter/ publications/pm_scm.pdf

Gunasekaran, Angappa; Williams, H. James \& McCaughey, Ronald E. (2005). Performance Measurement and Costing System in New Enterprise. Technovation, 25, 523533. Disponible en: https://www.umassd. edu/media/umassdartmouth/businessinnovationresearchcenter/publications/pm newenterprise.pdf

Gustin, Craig M.; Daugherty, Patricia J. \& Stank, Theodore P. (1995). The Effects of Information Availability on Logistics Integration. Journal of Business Logistics, 16 (1), 1-21. 
Hälinen, Hanne-Mari (2015). Understanding the Concept of Logistic Cost in Manufacturing. Turku: Publications of Turku School of Economics. Disponible en: https://www. doria.fi/bitstream/handle/10024/103549/ Ae-1_2015.pdf?sequence $=2$

Heredia-Gutiérrez, Diobaldo César (2010). Metodología para implantar un sistema de costeo $\mathrm{ABC}$ a la industria de la confección. Revista Dictamen Libre, 7, 10-30. Disponible en: http://www.academia.edu/15854315/ Metodologia_ABC

Hicks, Douglas T. (1998). El sistema de costos basados en actividades ( $A B C)$. Guía para su implantación en pequeñas y medianas empresas. México: Alfaomega Grupo Editor.

Hilton, Ronald W. \& Platt, David E. (2005). Managerial Accounting: Creating Value in a Dynamic Business Environment. New York: McGraw-Hill/Irwin.

Ibusuki, Ugo \& Kaminski, Paulo Carlos (2007). Product Development Process with Focus on Value Engineering and Target Costing: A Case Study in an Automotive Company. International Journal of Production Economics, 105 (2), 459-474.

Innes, John \& Mitchell, Falconer (1997). The Application of Activity-Based Costing in the United Kingdom's Largest Financial Institutions. The Service Industries Journal, 17 (1), 190-203.

Institute of Management Accountants (1994). Implementing Target Costing. Disponible en: http://www.imanet.org/PDFs/Public/ Research/SMA/Implementing\%20 Target\%20Costing.pdf
Jariri, Fereydoon \& Zegordi, Seyed Hessameddin (2008). Quality Function Deployment, Value Engineering and Target Costing, an Integrated Framework in Design Cost Management: A Mathematical Programming Approach. Scientia Iranica, 15 (3), 405-411.

Jiang, $\mathrm{Su}$ (2013). Total Cost of Ownership Analysis between D.G. Hybrid and Solar Energy Power Supply Systems for BTS. Proceedings of $35^{\text {th }}$ International Telecommunications Energy Conference Smart Power and Efficiency, INTELEC. Hamburg, Germany. Kaplan, Robert S. \& Anderson, Stephen R. (2004). Time-Driven Activity-Based Costing. Harvard Business Review, 1-18. Disponible en: https://hbr.org/2004/11/ time-driven-activity-based-costing

King, Margaret; Lapsley, Irvine \& Mitchell, Falconer (1994). Activity Based Costing in Hospitals: A Case Study Investigation. Chartered Institute of Management Accountants, CIMA.

Kirli, Mustafa \& Gümüş, Harun (2011). The Implementation of Strategic Management Accounting Based on Value Chain Analysis: Value Chain Accounting. International Journal of Social Sciences and Humanity Studies, 3 (1), 1-15. Disponible en: http://www.sobiad. org/ejournals/journal_ijss/arhieves/2011_1/ mustafa_kirli.pdf

Kivinen, Pasi \& Lukka, Anita (2004). Value Added Logistical Support Service: Logistics Cost Structure and Performance in the New Concept. Lappeenranta, Finland: Lappeenranta University of Technology, 
LUT. Disponible en: http://www.doria.fi/ handle/10024/31023

Kocakülâh, Mehmet C. \& Austill, A. David (2006). Product Development and Cost Management Using Target Costing: A Discussion and Case Analysis. Journal of Business \& Economics Research, JBER, 4 (2), 61-71. Disponible en: http://www. cluteinstitute.com/ojs/index.php/JBER/ article/view/2638/2684

Krajnc, Julijana; Logožar, Klavdij \& Korošec, Bojana (2011). Activity-Based Management of Logistic Costs in a Manufacturing Company. A Case of Increased Visibility of Logistic Costs in a Slovenian Paper Manufacturing Company. Promet-Traffic \& Transportation, 24 (1), 15-24. Disponible en: hrcak.srce.hr/file/122160

LaLonde, Bernard J. \& Pohlen, Terrance L. (1996). Issues in Supply Chain Costing. International Journal of Logistics Management, 7 (1), 1-12. Disponible en: http://citeseerx. ist.psu.edu/viewdoc/download?doi=10.1.1.1 97.5582\&rep $=$ rep $1 \&$ type $=$ pdf

Lambert, Douglas M.; Stock, James R. \& Ellram, Lisa M. (1998). Fundamentals of Logistics Management. Boston: Irwin/McGraw-Hill.

Li, Ling; Su, Qin \& Chen, Xu (2011). Ensuring Supply Chain Quality Performance through Applying the SCOR Model. International Journal of Production Research, 49 (1), 33-57. Lin, Binshan; Collins, James \& Su, Robert K. (2001). Supply Chain Costing: An ActivityBased Perspective. International Journal of Physical Distribution and Logistics Management, 31 (10), 702-713.
Lin, Man-Li (2009). Target Costing Practice of Strategic Outsourcing Partners: A Case Study of Sporting Goods Manufacturers. Service Operation, Logistics and Informatics, 639-644.

López-Mejía, María Rosa; Gómez-Martínez, Alicia \& Marín-Hernández, Salvador (2011). Sistema de Costos ABC en la mediana empresa industrial mexicana. Cuadernos de Contabilidad, 12 (30), 23-43. Disponible en: http://revistas.javeriana.edu.co/index. php/cuacont/article/view/3109/2283

Macharis, Cathy; Lebeau, Philippe; Mierlo, Joeri van \& Lebeau, Kenneth (2013). Electric versus Conventional Vehicles for Logistics: A Total Cost of Ownership. Electric Vehicle Symposium and Exhibition, EVS27. Barcelona.

Mageed, Ibrahim Abd El \& Kelety, Ali El (2006). Towards A Conceptual Framework For Strategic Cost Management: The Concept, Objectives, and Instruments. Chemnitz: Universidad Chemnitz de Tecnología. Disponible en: http://www.qucosa.de/ fileadmin/data/qucosa/documents/5228/ data/Title_250706.pdf

Mariana, Radu (2013). Modernizing Management Accounting by The ABC Method. Internal Auditing \& Risk Management, 8 (4), 5-16.

Marques, Maria da Conceição da Costa (2012). Contribución del modelo $\mathrm{ABC}$ en la toma de decisiones: el caso universidades. Cuadernos de Contabilidad, 13 (33), 527543. Disponible en: http://revistas. javeriana.edu.co/index.php/cuacont/article/ view/4282/3251 
Mauleón-Torres, Mikel (2006). Logística y costos. Madrid: Ediciones Díaz de Santos.

Mena, Carlos; Whicker, Linda; Templar, Simon \& Bernon, Michael (2002). Costing the Supply Chain. Manufacturing Engineer, 81 (5), 225-228.

Miller, Jeffrey G. \& Vollmann, Thomas E. (1985). The Hidden Factory. Harvard Business Review, 63 (5), 142-150. Disponible en: https://hbr.org/1985/09/the-hidden-factory

Monden, Yasuhiro \& Hamada, Kasuki (1991). Target Costing and Kaizen Costing in Japanese Automobile Companies. Journal of Management Accounting Research, 3, 1634. Disponible en: http://www.ftms.edu. my/images/Document/BB315009S\%20 -\%20Strategic\%20Management\%20 Accounting/Monden,Hamada-1991Targetcostingandkaizencosting.pdf Noreen, Eric (1991). Conditions under which Activity-Based Costing Systems Provide Relevant Costs. Journal of Management Accounting Research, 3 (4), 159-168. Disponible en: https://fisher.osu. edu/ young.53/Noreen\%20JMAR\%201991 Öker, Figen \& Adigüzel, Hümeyra (2010). Time-Driven Activity-Based Costing: An Implementation in a Manufacturing Company. Journal of Corporate Accounting \& Finance, 22 (1), 75-92.

Orjuela-Castro, Javier Arturo \& Adarme-Jaimes, Wilson (2014). Identificación de asimetrías en los medios de almacenamiento y transporte en la cadena de frutas colombiana. Primer Congreso Internacional Industria y Organizaciones, Logística para la Competitividad. Bogotá.
Orjuela-Castro, Javier Arturo; Castañeda-Calderón, Camilo Andrés \& Calderón, María Eugenia (2011). Análisis de la cadena de valor en las estructuras productivas de uchuva y tomate de árbol en la provincia de Sumapaz y el Distrito Capital. Ingeniería, Revista Científica y Tecnológica de la Facultad de Ingeniería de la Universidad Distrital Francisco José de Caldas, 13 (2), 4-12. Disponible en: http://revistas.udistrital.edu.co/ojs/index. php/reving/article/view/2663/3818

Orjuela-Castro, Javier Arturo; CastañedaMoreno, Ibeth Johana; Canal-Roa, Jorge Luis \& Rivera-Velasco, Jesús (2015). La logística en la cadena de frutas. Frutas $y$ Hortalizas, 39, 10-15. Disponible en: http:// www.asohofrucol.com.co/archivos/Revista/ Revista39.pdf

Parra-Ortega, Óscar Javier (2010). Componentes de costo en los modelos de diseño de cadenas de abastecimiento. Poliantea, 10, 201-207. Disponible en: http://journal.poligran.edu.co/index.php/ poliantea/article/view/237/217

Pau i Cos, Jordi \& Navascués y Gasca, Ricardo de (1998). Manual de logística integral. Madrid: Ediciones Díaz de Santos S.A.

Peppard, Joe \& Rylander, Anna (2006). From Value Chain to Value Network: Insights for Mobile Operators. European Management Journal, 24 (2-3), 128-141. Disponible en: https://www.researchgate. net/file.PostFileLoader.html?id $=551110$ 7bd039b1fe728b4600\&assetKey $=$ AS\% 3A273742171705360\%401442276627562

Petterson, Annelie I. \& Segerstedt, Anders (2012). Measuring Supply Chain Cost. In- 
ternational Journal of Production Economics, 143 (2), 357-363.

Pirttilä, Timo \& Hautaniemi, Petri (1995). Activity-Based Costing and Distribution Logistics Management. International Journal of Production Economics, 41 (1), 327-333.

Pohlen, Terrance L. \& LaLonde, Bernard J. (1994). Implementing Activity-Based Costing $(\mathrm{ABC})$ in Logistics. Journal of Business Logistics, 15 (2), 1-23.

Porter, Michael E. (1990). New Global Strategies for Competitive Advantage. Planning Review, 18 (3), 4-14.

Quiñónez, Diana; González, Nora; López, María Elvira \& Tabares, María Guadalupe (2005). Diferencia entre el costeo tradicional y el costeo basado en actividades. $E l$ Buzón de Pacioli, Revista del Departamento de Contaduría y Finanzas del Instituto Tecnológico de Sonora. Disponible en: http://www. itson.mx/publicaciones/pacioli/Documents/ no60/costo.pdf

Radziwill, Nicole \& DuPlain, Ronald F. (2010). Using Value-Based Total Cost of Ownership (TCO) Measures to Inform Subsystem Trade-Offs. Proceedings of SPIE - The International Society for Optical Engineering, SPIE Astronomical Telescopes + Instrumentation, 77380W-77380W-10. Disponible en: https://www.researchgate. net/publication/253829455_Using_ValueBased_Total_Cost_of_Ownership_TCO_ Measures_to_Inform_Subsystem_Tradeoffs

Ritsma, Roel J.; Tuyl, André \& Snijders, Bas (2009). Buying the Lowest Total Cost of Ownership (TCO). Petroleum, Chemical and Related Process Industries, PCIC Europe, 2009. Conference Record. Barcelona. Disponible en: http://www.eaton.eu/ecm/ idcplg?IdcService=GET_FILE\&allowInterr upt $=1 \&$ RevisionSelectionMethod $=$ LatestR eleased $\&$ noSaveAs $=0 \&$ Rendition $=$ Primary \&dDocName $=$ PCT_358291

Robeson, James F. \& Copacino, William C. (eds.) (1994). The Logistics Handbook. New York: Andersen Consulting.

Rof, Maria Letiţia \& Farcane, Nicoleta (2011). Current State and Evolution Perspectives for Management Accounting in the Energy Sector by Implementing the ABC Method. Conference Procedings. European IntegrationNew Challenges, 653-660. Oradea, Romania. Disponible en: https://www. researchgate.net/publication/227462805_ CURRENT_STATE_AND_EVOLUTION_ PERSPECTIVES_FOR_MANAGEMENT_ ACCOUNTING_IN_THE_ENERGY_ SECTOR_BY_IMPLEMENTING_THE_ ABC_METHOD

Ruiz de Arbulo-López, Patxi; Fortuny-Santos, Jordi; Vintró-Sánchez, Carla \& BasáñezLlantada, Aitor (2013). Aplicación de time-driven activity-based costing en la producción de componentes de automóvil. Dyna, 88 (2), 234-240. Disponible en: http://www.revistadyna.com/documentos/ pdfs/_adic/5105-2.pdf

Ruiz-Moreno, Andrés Felipe; Caicedo-Otavo, Andrés Leonardo \& Orjuela-Castro, Javier Arturo (2015). Integración externa en las cadenas de suministro agroindustriales: una revisión al estado del arte. Ingeniería, Revista Científica y Tecnológica de la Facultad de 
Ingeniería de la Universidad Distrital Francisco José de Caldas, 20 (2), 167-188. Disponible en: http://revistas.udistrital.edu.co/ojs/ index.php/reving/article/view/8278/10759

Sachan, Amit; Sahay, B. S. \& Sharma, Dinesh (2005). Developing Indian Grain Supply Chain Cost Model: A System Dynamics Approach. International Journal of Productivity and Performance Management, 54 (3), 187205.

Sahin, Funda \& Robinson, Powell Ersell (2005). Information Sharing and Coordination in Make-to-Order Supply Chains. Journal of Operation Management, 23 (6), 579-598. Disponible en: http://www.bauer. uh.edu/doctoral/scm/docs/Sahin-2.pdf

Salazar-Sanabria, Hugo Felipe \& LópezBello, César Amílcar (2009). Propuesta metodológica para la aplicación del modelo Supply Chain Operations Reference SCOR. Ingeniería, Revista Científica y Tecnológica de la Facultad de Ingeniería de la Universidad Distrital Francisco José de Caldas, 14 (2), 3441. Disponible en: http://revistas.udistrital. edu.co/ojs/index.php/reving/article/ view/2377/3257

Sani, Alireza Azimi \& Allahverdizadeh, Mahdi (2012). Target and Kaizen Costing. World Academy of Science, Engineering and Technology, 6, 40-46. Disponible en: http://waset.org/publications/11016/ target-and-kaizen-costing, http://www. internationalscienceconference.org/ publication/11016

Schulze, Manuel; Seuring, Stefan \& Ewering, Christian (2012). Applying Activity-Based Costing in a Supply Chain Environment. In- ternational Journal of Production Economics, 135 (2), 716-725.

Setyawati, Dian R.; Santoso, Imam \& Effendi, Mas'ud (2012). Value Chain Analysis on the Logistics Management as the Basis for Strategy Formulation to Increase Customer Satisfaction (Case Study in PT Coca-Cola Amatil Indonesia - Plant East Java). Agroindustrial Journal, 1 (1), 28-35. Disponible en: http://www.apta.or.id/index. php/agroindustrial/article/view/41

Seuring, Stefan (2002). Cost Management in Supply Chain-Different Research Approaches. En Stefan Seuring \& Maria Goldbach (eds.). Cost Management in Supply Chain, 1-11. Heidelberg: Physica-Verlag.

Shank, John K. \& Govindarajan, Vijay (1992).

Strategic Cost Analysis of Technology Investments. MIT Sloan Management Review, 34 (1), 39-51.

Shank, John K. \& Govindarajan, Vijay (1993). Strategic Cost Management: The New Tool for Competitive Advantage. New York: The Free Press.

Sharaf-Addin, Hussein H.; Omar, Normah \& Sulaiman, Suzana (2014). Target Costing Evolution: A Review of the Literature from IFAC's (1998) Perspective Model. Canadian Center of Science and Education, 10 (9), 82-99. Disponible en: http:// ccsenet.org/journal/index.php/ass/article/ download/36575/20561

Sinisterra-Valencia, Gonzalo \& Polanco-Izquierdo, Luis Enrique (2007). Contabilidad administrativa. Bogotá: ECOE Ediciones.

Smith, Wilbur I.; Lewis, Gabrielle; Churchwell, Tremika \& Benjamin, Colin (2002). In- 
tegrating Activity-Based Costing, Target Costing and Value Engineering for Supply Chain Improvement. Proceedings of the $5^{\text {th }}$ Biannual World Automation Congress, Tallahassee, Florida.

Somapa, Sirirat; Cools, Martine \& Dullaert, Wout (2010). Time-Driven Activity-Based Costing in a Small Road Transport and Logistics Company. Proceedings of the $2^{\text {nd }}$ International Conference on Logistics and Transport \& the $1^{\text {st }}$ International Conference on Business and Economics. Queenstown, New Zealand. Disponible en: http://preet. sesolution.com/iclt2010/Full\%20Papers/ Distribution,\%20Transportation,\%20 and\%20Traffic/0056-Sirirat.pdf

Sople, Vinod V. (2007). Logistics Management: The Supply Chain Imperative. Delhi, India: Pearson Education.

Souissi, Mohsen \& Ito, Kazunoti (2004). Integrating Target Costing and the Balanced Scorecard. The Journal of Corporate Accounting \& Finance, 15 (6), 57-62. http://dx.doi. org/10.1002/jcaf.20057

Suárez-Tirado, Jorge (2013). Control de gestión en la cadena de valor y los aportes de la contabilidad de gestión: estudio de caso de una compañía colombiana. Cuadernos de Contabilidad, 14 (34), 245-261. Disponible en: http://cuadernosdecontabilidad. javeriana.edu.co/vol14_n_34/vol14_34_9. pdf

Themido, Isabel Hall; Arantes, Amílcar; Fernandes, Carla \& Guedes, A. Paulo (2000). Logistic Costs Case Study - An ABC Approach. Journal of the Operational Research Society, 51 (10), 1148-1157.
Tseng, Yung-yu; Yue, Wen Long \& Taylor, Michael (2005). The Role of Transportation in Logistics Chain. Proceedings of the Eastern Asia Society for Transportation Studies, 5, 1657-1672. Disponible en: https://www. siam.org/journals/plagiary/1657.pdf

Urrea-Bello, Luis Martín; Garzón-Cárdenas, Luz Gabina \& Pérez-Suárez, Lucía Elena (2007). Medición del desempeño en la cadena de abastecimiento del sector floricultor colombiano. Activos, Revista de la Facultad de Contaduría Pública, 7 (13), 15-46. Disponible en: http://revistas. usta.edu.co/index.php/activos/article/ view/2747/2656

Walker, Mike (1998). Attributes or Activities? Looking to ABCII. Australian CPA, 68, 2628.

Weiyi, Fu \& Luming, Yang (2009). The Discussion of Target Cost Method in Logistics Cost Management. 2009 ISECS International Colloquium on Computing, Communication, Control \& Management, 537-540.

Wouters, Marc; Anderson, James C. \& Wynstra, Finn (2005). The Adoption of Total Cost of Ownership for Sourcing Decisions: A Structural Equations Analysis. Accounting, Organisations and Society, 30 (2), 167-191.

Wright, Wilmer (1962). Direct Standard Costs for Decision Making and Control. New York: McGraw-Hill.

Wu, Claudia (2005). Total Supply Chain Cost Model. Thesis, Massachussets Institute Technology, 1-74. Disponible en: https://dspace.mit.edu/bitstream/ handle/1721.1/34869/64560808-MIT. pdf?sequence $=2$ 
Xiaoling, Ye (2011). Logistics Cost Management Based on ABC and EVA Integrated Model. Proceedings International Conference on Automation and Logistics, 262-266. Chongqing.

Zachariassen, Frederik \& Stentoft, Jan (2011). Total Cost of Ownership: A Differentiated Approach. Industrial Management \& Data Systems, 111 (3), 448-469.

Zakariah, Sahidah \& Pyeman, Jaafar (2013). Logistics Cost Accounting and Management in Malaysia: Current State and Challenge. International Journal of Trade, Economics and Finance, 4 (3), 119-123. Disponible en: http:// www.ijtef.org/papers/270-CF3008.pdf

Zang, Jing; Zhang, Keming Z. \& Zhao, Rui (2008). Application of Activity-Based Costing to the Logistics Cost Management of Agricultural Products. IEEE International Conference on Service Operations and Logistics, and Informatics.

Zeng, Amy Z. \& Rossetti, Christian (2003). Developing a Framework for Evaluating the Logistics Costs in Global Sourcing Processes. International Journal of Physical Distribution \& Logistics Management, 33 (9), 785-803.

Zhang, Guo-qing \& Liu, Long-qing (2009). A Study on the Calculable Method about Business Logistics Cost. International Conference on Management and Service Science. Wuhan.

Zheng, Peng \& Wang, Man (2011). Application of the Activity-Based Costing for Third-
Party Logistics Companies. Business Management and Electronic Information, BMEI, 2, 346-349.

Zokaei, Keivan (2007). Value Chain Analysis. En Hossein Bidgoli (ed.). The Handbook of Technology Management. Supply Chain Management, Marketing and Advertising, and Global Management, Vol. 2, 1-15. Hoboken, New Jersey: Wiley \& Sons.

Zokaei, Keivan \& Simons, David W. (2006).

Performance Improvements through Implementation of Lean Practices: A Study of the UK Red Meat Industry. International Food and Agribusiness Management Review, 9 (2), 30-53. Disponible en: http://www. ifama.org/resources/Documents/v9i2/ Zokaei-Simmons.pdf

- Fecha de recepción: 23 de marzo de 2016

- Fecha de aceptación: 20 de junio de 2016

- Disponible en línea: 14 de diciembre de 2016

\section{Para citar este artículo}

Orjuela-Castro, Javier Arturo; Suárez-Camelo, Norberto \& Chinchilla-Ospina, Yamit Israel (2016). Costos logísticos y metodologías para el costeo en cadenas de suministro: una revisión de la literatura. Cuadernos de Contabilidad, 17 (44), 377420. https://dx.doi.org/10.11144/Javeriana.cc17-44.clmc 